\title{
Robust Optimal Control for Demand Side Management of Multi- Carrier Microgrids
}

This paper was downloaded from TechRxiv (https://www.techrxiv.org).

LICENSE

CC BY-NC-SA 4.0

SUBMISSION DATE / POSTED DATE

09-02-2022 / 11-02-2022

\section{CITATION}

Carli, Raffaele; Cavone, Graziana; Pippia, Tomás; De Schutter, Bart; Dotoli, Mariagrazia (2022): Robust Optimal Control for Demand Side Management of Multi-Carrier Microgrids. TechRxiv. Preprint. https://doi.org/10.36227/techrxiv.19149023.v1

$\mathrm{DOI}$

10.36227/techrxiv.19149023.v1 


\title{
Robust Optimal Control for Demand Side Management of Multi-Carrier Microgrids
}

\author{
Raffaele Carli ${ }^{1}$, Member, IEEE, Graziana Cavone ${ }^{1}$, Member, IEEE, Tomás Pippia ${ }^{2}$, \\ Bart De Schutter ${ }^{2}$, Fellow, IEEE, and Mariagrazia Dotoli ${ }^{1}$, Senior Member, IEEE
}

\begin{abstract}
This paper focuses on the control of microgrids where both gas and electricity are provided to the final customer, i.e., multi-carrier microgrids. Hence, these microgrids include thermal and electrical loads, renewable energy sources, energy storage systems, heat pumps, and combined heat and power units. The parameters characterizing the multi-carrier microgrid are subject to several disturbances, such as fluctuations in the provision of renewable energy, variability in the electrical and thermal demand, and uncertainties in the electricity and gas pricing. With the aim of accounting for the data uncertainties in the microgrid, we propose a Robust Model Predictive Control (RMPC) approach whose goal is to minimize the total economical cost, while satisfying comfort and energy requests of the final users. In the related literature various RMPC approaches have been proposed, focusing either on electrical or on thermal microgrids. Only a few contributions have addressed the robust control of multi-carrier microgrids. Consequently, we propose an innovative RMPC algorithm that employs on an uncertainty set-based method and that can provide better performance compared with deterministic model predictive controllers applied to multi-carrier microgrids. With the aim of mitigating the conservativeness of the approach, we define suitable robustness factors and we investigate the effects of such factors on the robustness of the solution against variations of the uncertain parameters. We show the effectiveness of the proposed RMPC approach by applying it to a realistic residential multi-carrier microgrid and comparing the obtained results with the ones of a baseline robust method.
\end{abstract}

Note to Practitioners-This work is motivated by the emerging need for effective energy management approaches in multicarrier microgrids. The inherent difficulty of scheduling simultaneously the operations of various energy infrastructures (e.g., electricity, natural gas) is exacerbated by the inevitable presence of uncertainties that affect the inter-dependent dynamics of different energy resources and equipment. The proposed robust MPC-based control strategy allows the energy manager to effectively determine an optimal energy scheduling of multifaceted system components, making a tradeoff between performance and protection against data uncertainty. The presented strategy is comprehensive and generic, as it can be applied to different microgrid frameworks integrating various types of system components and sources of uncertainty, while at the same

1 R. Carli, G. Cavone, and M. Dotoli are with the Dept. of Electrical and Information Engineering, Polytechnic of Bari, Bari, Italy (email: \{raffaele.carli, graziana.cavone, mariagrazia.dotoli\}@poliba.it).

2 T. Pippia and B. De Schutter are with the Delft Center for Systems and Control, Delft University of Technology, Delft, The Netherlands (email: $\{$ t.m.pippia, b.deschutter\}@tudelft.nl).

This work received funding from the Italian University and Research Ministry under project Pico\&Pro (National Research Program, contract No. ARS01_01061) and in part by the Apulia Region (Italy) through the Research for Innovation (REFIN) Call under Project no. C9A3735B. time being implementable in any energy management system.

Index Terms-Energy and environment-aware automation, demand side management (DSM), multi-carrier microgrid, setbased uncertainty, robust optimization; robust model predictive control.

\section{INTRODUCTION}

We are currently facing an energy transition worldwide that has led to several changes in energy networks [1]. Due to technological advancements, governmental policies, and a larger share of renewables, electrical and thermal energy grids are changing, facilitating the introduction of new energy paradigms but at the same time creating many challenges. One of such paradigms is the introduction of microgrids, which, albeit an old concept, provide many benefits to the electrical grids [2]. Microgrids are energy grids of small size, including local production of energy, consumption, energy storage systems, a control architecture, and a connection to the main grid [3], [4]. Some of the benefits of microgrids are: increased efficiency, because the energy locally produced is also consumed locally, thus wasting less energy in transportation; higher resiliency, because the failure of a single microgrid does not compromise the stability of the whole grid; a modular structure, which allows more flexibility, so that each microgrid is adapted to the local characteristics of the region where it is located, e.g., renewable energy production from the sun or wind, or both. However, the introduction of a larger share of renewable energy into the grid, together with a higher amount of energy storage and variable electricity prices, which typically characterize microgrids, adds a layer of uncertainty that leaves many open challenges [5], [6].

In fact, in microgrid energy management problems, the main goal is to minimize an economical objective while providing good performance for other goals, e.g., minimizing the amount of energy exchanged with the main grid to avoid penalties as in [7], or the discomfort of occupants in a building [8]. Among several control algorithms presented in the literature, Model Predictive Control (MPC) [9], [10] stands out as one of the most suitable and flexible control approaches for the problem to face. MPC is a model-based, optimization-based control approach that allows including several objectives and constraints in the formulation of the control problem. In the standard deterministic MPC (Det-MPC) form, the unknown disturbances, e.g., electrical loads, renewable generation, are simply forecast with a point forecasting method. Given that the actual value of these perturbations can be quite different from the forecast itself, this can lead to poor performance. 
To cope with this issue, two different MPC approaches have been developed, namely stochastic and robust MPC (RMPC) [10]. On the one hand, in stochastic MPC, as the name mentions, the stochastic properties of the disturbances are exploited and used in such a way that the constraints of the problem are not satisfied for every possible disturbance realization but only for a subset of them. Such a strategy works better when there is a large amount of prior data available and the stochastic properties of the disturbances can be assumed to be known. On the other hand, RMPC generally satisfies the constraints of the problem for every possible realization of the disturbances. The only information needed in such a case is the disturbance bounds, which can be computed or estimated for a large variety of practical applications. Clearly, the choice of either method depends on the specific application. Where it is not vital to guarantee constraint satisfaction at all times, e.g., for building heating control [8], [11], a stochastic approach results in the best choice, to avoid a possibly too conservative control action. Conversely, when a small amount of information is available on the disturbances, an RMPC approach is the most suitable choice. This also applies to cases in which it is necessary to guarantee at all times a maximum amount of power exchanged with the main grid [12]. In addition, the robust approach has significantly higher computational performance and the corresponding uncertainty modeling is simpler than the stochastic approach [13]. For the above reasons, in this paper we focus on RMPC, and specifically on a robust control approach that can guarantee an adequate constraint satisfaction while not overly affecting the optimality of the resulting control strategies.

\section{A. Related works}

In the last years, many works have considered applications of MPC to microgrids [12], [14]-[27]. These works devote their attention either to multi-carrier microgrids [12], [16][22], to fully thermal microgrids [15], or to fully electrical microgrids [14], [23]-[27]. The article [14] presents a modeling framework for electrical microgrid energy management systems. Similarly, paper [15] presents a thermal microgrid modeling framework and a Det-MPC approach, considering district heating, thermal energy storage, and flexible loads. MPC algorithms for multi-carrier microgrids have been proposed in [12], [16]-[19], [22]. The article [16] discusses a stochastic MPC approach for microgrids that contain both thermal and electrical units. The control approach uses a twostage optimization strategy: in the first stage, a decision on the microgrid operations is made before the values of the external disturbances are known; in the second stage, after the values of the random variables become known, correction actions are taken. The authors of [17] develop an MPC approach for a multi-carrier energy management system of a microgrid. Stability of the controlled system is proven, although the resulting controller is deterministic and does not take disturbances into account. Paper [18] considers a demand response framework, also within the context of Det-MPC. In [19], similarly to [16], a two-stage MPC controller for mixed-energy microgrids is presented, where, however, the first stage controller consists of a stochastic MPC algorithm while the second stage is a rule-based one. Similarly to [16], a two-stage optimization procedure is proposed in [22] for multi-carrier microgrids. In the first stage, a day-ahead scheduling is performed, while in the second stage corrective actions are taken with a shrinking receding horizon approach. The resulting MPC controller is deterministic, as disturbances are simply point-forecasts.

With respect to RMPC, some applications to microgrids have been considered [12], [22]-[26]. The authors of [23] present a min-max RMPC approach for fuel-cell cars inside a microgrid. Such vehicles can be used as a power generating unit when they are parked inside the microgrid and not being used. In [24], an economic RMPC controller is presented. Constraints related to the operational limits of the components of the microgrid and on the energy balance are included in the controller, considering variations of the expected loads and using a constraint tightening strategy. The constraints on the states and inputs are tightened and the resulting control law guarantees convergence to a neighborhood of a robust optimal trajectory that minimizes the cost function. In [25] an RMPC method, which has three types of uncertainty scenarios and uncertainty budgets for islanded microgrids, is presented. In [27], a hierarchical controller is presented, in which the lower level comprises a rule-based controller while the upper level consists of an RMPC controller that considers a robust optimization over a control policy parameterized by gains and that is able to compensate uncertainties on predictions of loads, which are modeled based on fuzzy intervals. The authors of [26] consider an RMPC approach for islanded electrical microgrids, where the "degree of uncertainty" method of [28] is used. Such a method provides a trade-off between robustness and conservatism of the controller. In [12], an RMPC approach for multi-carrier microgrids is considered, with a focus on a demand response program of the United Kingdom national grid. The goal of such a program is to add some extra flexibility to the energy grid and make the supply of energy more secure; the prosumer that provides flexibility is rewarded economically for the commitment to supply energy. A nonlinear economical MPC approach is presented in [22]. The authors focus on the control of combined-heat-and-power plant that provides electrical and thermal energy to a district and they assume the existence of a thermal market. To improve the robustness of the controller, a simple approach is adopted, in which three different scenarios are forecast, i.e. expected and upper and lower extreme scenario. Then, a weighted sum of the cost function, for the three scenarios, is used as objective.

\section{B. Contributions}

It can be noted from the previous literature review that, among the papers that present an RMPC algorithm, i.e., [12], [22]-[26], almost all of them actually consider only a fully electrical microgrid that does not include any thermal device. The papers that do consider a multi-carrier microgrid, i.e. [12], [22], have a limited scope. In fact, [12] focuses on the short-term operating reserve in the national context of the United Kingdom, while [22] considers a single device, i.e. a 
combined-heat-and-power plant, and it assumes the presence of a thermal power exchange grid.

Therefore, to the best of the authors' knowledge, little or no attention has been devoted in the literature to RMPC algorithms for microgrids that consider both electricity and heating devices. Most of the works focus either on an RMPC strategy for single-carrier microgrids or on a multi-carrier microgrid with a Det-MPC algorithm. However, heating systems are becoming increasingly important, as more and more devices that couple the heating demand with the electrical one are installed in buildings, e.g. heat pumps (HPs), micro combined-heatand-power plants ( $\mu$-CHPs). Moreover, as mentioned earlier, the large amount of uncertainties in the microgrid, related to energy demand and supply, results in the need for adopting an RMPC strategy.

Therefore, in this work we address the robust energy management system problem of a multi-carrier microgrid that includes both electrical and thermal devices. In particular, on the heating side, we consider HPs, $\mu$-CHPs, controllable thermal loads (CTLs), non-controllable thermal loads (NCTLs), thermal energy storage systems (TESSs), and auxiliary boilers, while on the electrical side we consider non-controllable electrical loads (NCELs), controllable electrical loads (CELs), electrical energy storage systems (EESSs), and renewable energy sources (RESs). Moreover, we develop a novel RMPC algorithm that provides improved performance with respect to Det-MPC algorithms present in the current literature. In order to achieve such a result, we adopt the cardinality-uncertaintyset robust optimization method proposed in [28], in which the so-called budgets of uncertainty are employed to control the degree of conservatism and robustness of the solution. We manage to achieve constraint satisfaction successfully even with large disturbances affecting both the demand and supply of energy within the electrical and thermal parts of the microgrid, as shown in the simulation results. Lastly, while we recently published a conference paper [20] with preliminary results, the current work significantly extends and improves the method in several aspects: 1) we extend the proposed microgrid model in order to integrate also further important components, e.g. controllable thermal loads; 2) we consider and model further sources of uncertainty acting in the microgrid, e.g. electricity and gas pricing; 3 ) we adopt the cardinality-set-uncertainty to define the uncertainty space for the online robust optimization; 4) we thoroughly compare the results achieved by the novel proposed method with a related robust approach.

Our contribution is therefore threefold:

- we present a novel mathematical model and a comprehensive RMPC methodology to optimally control the energy exchange of a multi-carrier microgrid equipped with both thermal and electrical units, namely thermal loads, electrical loads, renewable energy sources, energy storage systems, heat pumps, and combined heat and power units.

- we take into account the data uncertainty associated with electrical and thermal energy demand, RES generation, and electricity and natural gas coefficients in the microgrid by formulating a computationally tractable robust counterpart of the online energy scheduling problem based on an uncertainty set-based method of [28].

- we define several suitable robustness factors to mitigate and reduce the conservativeness of the proposed approach. Moreover, we investigate the effects of such factors on the robustness of the solution against variations of the uncertain parameters within the given uncertainty sets.

\section{Outline}

The outline of the article remainder is as follows. In Section II, we discuss the system model. Section III is focused on the Det-MPC formulation. We present the RMPC energy scheduling approach in Section IV, highlighting the difference with respect to the deterministic approach and the definition of data uncertainty set. Section V is devoted to numerical experiments, showing the effectiveness of the proposed robust control scheme and comparing the achieved results with a related robust approach. Lastly, conclusions and remarks for future work are presented in Section VI.

\section{Model of The Multi-CARrier Microgrid}

The multi-carrier microgrid reported in this section is based on the model shown in Fig. 1. The main electrical grid can supply energy to CELs, NCELs, and HP (or to CELs, NCELs, $\mathrm{HP}$, and EESS), while it can receive energy from RES, EESS, and CHP (or RES and CHP). Consequently, the system can both buy and sell energy to the main grid. The electrical demands can be fulfilled by the main electrical grid, RES, CHP, and EESS. On the other hand, the gas network can supply the CHP and the boiler, while it cannot receive gas from the microgrid. The thermal energy produced by CHP, boiler, and HP (or by CHP, boiler, HP, and TESS) can be provided to the NCTL, CTL, and TESS (or to NCTL, CTL).

We remark that the considered microgrid architecture in Fig. 1 is quite general and represents the majority of practically employed multi-carrier microgrids. Specifically, the scheme in Fig. 1 does not represent the actual topology of the gas and the electrical grid (which incorporates several buses), but rather it constitutes a high-level conceptual model of the addressed microgrid, aimed at synthetically showing the main inflows/outflows of the considered components.

In the sequel we consider a control horizon $[k+1, k+H]$ containing $H$ equally spaced time slots and moving ahead at each current time slot $k$.

\section{A. Notation}

Let $\mathbb{N}, \mathbb{R}, \mathbb{Z}_{+}, \mathbb{R}^{n}, \mathbb{R}^{n \times m}$ denote the set of natural numbers, the set of real numbers, the set of non-negative integers, the set of column real vectors of length $n$ and the set of $n$ by $m$ real matrices, respectively. The transpose of a matrix $A \in \mathbb{R}^{n \times m}$ is denoted by $A^{\top}$, while $\mathbf{1}_{n}$ and $\mathbf{0}_{n}$ denotes a column vector of $n$ elements all being equal to 1 and 0 , respectively. $\circ$ denotes the entry-wise product between two equal size vectors. $\lfloor\cdot\rfloor$ denotes the ceiling operator: given the real number $a,\lfloor a\rfloor$ is the greatest integer lower than or equal to a. For any $k_{1}, k_{2} \in \mathbb{N}$ 


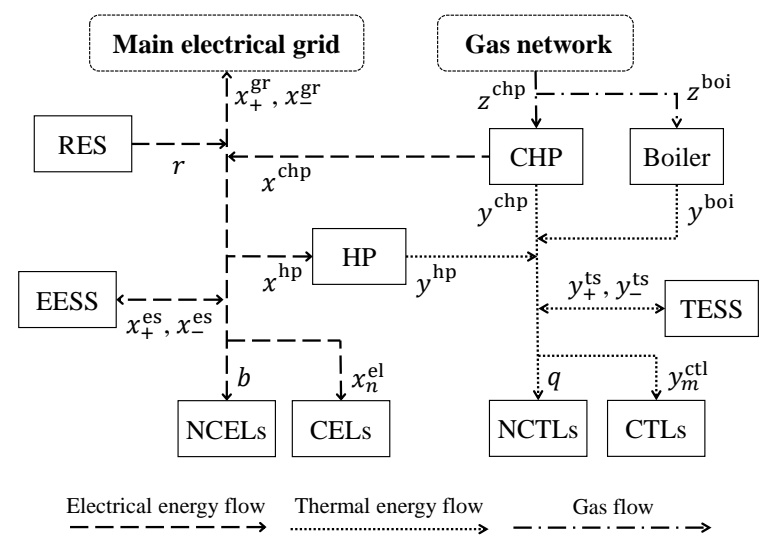

Fig. 1. High-level scheme of the multi-carrier microgrid illustrating the considered components and the corresponding inflows and outflows.

with $k_{1} \leq k_{2}$, the finite set of integers $\left\{k_{1}, \ldots, k_{2}\right\}$ is denoted by $\mathbb{N}_{\left[k_{1}, k_{2}\right]}$. For any $w_{1}, w_{2} \in \mathbb{Z}_{+}$with $w_{1} \leq w_{2},\left[w_{1}, w_{2}\right]$ denotes the closed interval $\left\{w \in \mathbb{Z}_{+} \mid w_{1} \leq w \leq w_{2}\right\}$. The vector $\mathbf{a}(k)=[a(k+1), \ldots, a(k+H)]^{\top}$ represents a column vector of $H$ values over the time horizon $[k+1, k+H]$. Lastly, $\operatorname{col}\left(\mathbf{a}_{n}\right)_{n \in \mathbb{N}_{[1, N]}}$ is equal to $\left(\mathbf{a}_{1}^{\top}, \ldots, \mathbf{a}_{N}^{\top}\right)^{\top}$.

\section{B. Electrical Loads}

The microgrid includes some NCELs for which the electrical demand cannot be shifted in time and regulated. At each time step $k \in \mathbb{Z}_{+}$let $\mathbf{b}(k)$ be a column vector of input parameters representing the energy consumption profile of the NCELs over the time horizon $[k+1, k+H]$. Moreover, the grid comprises $N^{\text {cel }}$ CELs that allow for operations' regulation and programming. The decision variables representing the energy consumption of each CEL over the time horizon $[k+1, k+H]$ is represented by the column vector $\mathbf{x}_{n}^{\mathrm{el}}(k)$ with $n \in \mathbb{N}_{\left[1, N^{\text {cel }}\right]}$. We collect the profiles of all CELs in the column vector $\mathbf{x}^{\mathrm{el}}(k):=\operatorname{col}\left(\mathbf{x}_{n}^{\mathrm{el}}(k)\right)_{n \in \mathbb{N}_{\left[1, N^{\text {cel }}\right.}}$.

Differently from NCELs, CELs are required to work in bounded operating intervals limited by minimum and maximum operating levels. The maximum and the minimum energy levels for each CEL are respectively represented by the input parameters vectors $\overline{\mathbf{l}}_{n}(k)$ and $\underline{\mathbf{l}}_{n}(k)$. Moreover, each $n$ CEL must consume a well-defined quantity of energy $L_{n}(k)$ over the considered time horizon. The defined requirements can be summarized by the following constraints:

$$
\begin{gathered}
\underline{\mathbf{l}}_{n}(k) \leq \mathbf{x}_{n}^{\mathrm{el}}(k) \leq \overline{\mathbf{l}}_{n}(k), n \in \mathbb{N}_{\left[1, N^{\mathrm{cel}}\right]} \\
\mathbf{1}_{H}^{\top} \mathbf{x}_{n}^{\mathrm{el}}(k)=L_{n}(k), n \in \mathbb{N}_{\left[1, N^{\mathrm{cel}}\right]} .
\end{gathered}
$$

\section{Thermal Loads}

Similarly to electrical loads, thermal loads are distinguished into NCTLs and CTLs, and take into account the thermal demand, e.g., for heating space and water. As regards NCTLs, e.g., the thermal demand for heating water, the consumption profile over the time horizon $[k+1, k+H]$ is represented by a vector of input parameters $\mathbf{q}(k)$. Differently, the $M^{\text {ctl }}$ CTLs consumption profiles, which can be programmed and shifted over time, e.g., the space heating/cooling, is defined as a column vector of decision variables $\mathbf{y}_{m}^{\text {ctl }}(k)$ with $m \in \mathbb{N}_{\left[1, M^{\text {ctl }}\right]}$. CTLs are requested to work fulfilling comfort requirements in a bounded interval limited by minimum and maximum thermal levels. In particular, the maximum and minimum temperature profiles for each CTL are respectively represented as $\overline{\mathbf{T}}_{m}(k)$ and $\underline{\mathbf{T}}_{m}(k)$. A CTL is formalized as first order dynamic system function of the temperature of the environment and of the related decision variables. The defined assumptions can be formulated as follows:

$$
\begin{gathered}
\underline{\mathbf{T}}_{m}(k) \leq \mathbf{T}_{m}(k) \leq \overline{\mathbf{T}}_{m}(k), m \in \mathbb{N}_{\left[1, M^{\mathrm{ct}}\right]} \\
\mathbf{T}_{m}(k)=e^{-\frac{\Delta \mathrm{h}}{\tau}} \mathbf{T}_{m}(k-1)+ \\
+\left(1-e^{-\frac{\Delta h}{\tau}}\right)\left(\mathbf{T}_{e}(k-1)+\alpha \mathbf{y}_{m}^{\mathrm{ctl}}(k)\right)
\end{gathered}
$$

where $\Delta \mathrm{h}$ is the sampling time of the time horizon $[k+1, k+$ $H], \tau$ is the constant time of the first-order dynamics of the environment temperature, $\alpha$ is the temperature gain of the heat exchanger, $\mathbf{T}_{e}(k-1)$ is the external temperature profile, and $\mathbf{y}_{m}^{\text {ctl }}(k)$ is the decision variables vector related to the $m$-th CTL. We collect the profiles of all CTELs in the column vector $\mathbf{y}^{\mathrm{ctl}}(k):=\operatorname{col}\left(\mathbf{y}_{m}^{\mathrm{ctl}}(k)\right)_{m \in \mathbb{N}_{\left[1, M^{\mathrm{ctl}}\right]}}$.

\section{Renewable Energy Source}

The microgrid under analysis includes a RES, which can be for instance a wind turbine for residential use and/or a photovoltaic panel. The corresponding energy generation profile over the horizon $[k+1, k+H]$ is represented by the column vector $\mathbf{r}(k)$.

\section{E. Heat Pump}

Various HPs can be used to accommodate the space heating and hot water demand. However, in this work we refer to air-to-water source HPs in heating mode only. In fact, such systems can be easily installed as a convenient retrofit solution in existing facilities, thus providing thermal energy for both space heating and hot water services [29]. The efficiency of the heat pump is measured by the so-called Coefficient of Performance (COP) that depends on the internal temperature of the residential unit and on the thermal gap between the internal and external temperature of the residential unit. The COP is here considered to assume a constant value over the horizon [30], [31]. The COP - denoted as $\eta^{\text {hp }}$ - relates the input electrical energy vector $\mathrm{x}^{\mathrm{hp}}(k)$ and the output thermal energy vector $\mathbf{y}^{\mathrm{hp}}(k)$ as follows:

$$
\mathbf{y}^{\mathrm{hp}}(k)=\eta^{\mathrm{hp}} \mathbf{x}^{\mathrm{hp}}(k) .
$$

Operational requirements impose to limit the thermal energy between minimum and maximum values $\underline{p}^{\text {hp }}$ and $\bar{p}^{\text {hp }}$ as follows:

$$
\underline{p}^{\mathrm{hp}} \mathbf{1}_{H} \leq \mathbf{y}^{\mathrm{hp}}(k) \leq \bar{p}^{\mathrm{hp}} \mathbf{1}_{H} .
$$

In addition, further constraints are imposed to limit the ramp up and ramp down of the HP generation in accordance with the upward and downward ramp rates $\rho^{\text {hp }}$ and $\bar{\rho}^{\text {hp }}$ :

$$
\underline{\rho}^{\mathrm{hp}} \leq y^{\mathrm{hp}}(h)-y^{\mathrm{hp}}(h-1) \leq \bar{\rho}^{\mathrm{hp}}, \forall h \in[k+1, k+H] .
$$




\section{F. Gas Boiler}

Auxiliary boilers are usually used in microgrids in order to meet thermal demand peaks that cannot be satisfied by the CHP, HP, or TESS. In particular, gas-fired boilers are widely installed in facilities connected to the gas main grid and they are used to satisfy the heating demand when no decentralized power generation is available. The microgrid under analysis comprises a gas-fired boiler, which presents an efficiency ratio $\eta^{\text {boi }}$ around $100 \%$ during the burning process and can rapidly satisfy changes in the hot water demand thanks to its fast dynamics. The efficiency ratio $\eta^{\text {boi }}$ relates the input gas amount vector $\mathbf{z}^{\text {boi }}(k)$ and the output thermal energy vector $\mathbf{y}^{\text {boi }}(k)$ as follows:

$$
\mathbf{y}^{\text {boi }}(k)=\eta^{\text {boi }} \mathbf{z}^{\text {boi }}(k) .
$$

Operational requirements impose to limit the thermal energy generated by the gas boiler between minimum and maximum values $\underline{p}^{\text {boi }}$ and $\bar{p}^{\text {boi }}$ as follows:

$$
\underline{p}^{\text {boi }} \mathbf{1}_{H} \leq \mathbf{y}^{\text {boi }}(k) \leq \bar{p}^{\text {boi }} \mathbf{1}_{H} .
$$

\section{G. Combined Heat and Power Unit}

The microgrid comprises also a CHP unit that can generate both thermal and electrical energy using a single source of fuel. Complete CHP systems are usually deployed as a combination of a prime mover CHP technology, a TESS, and an auxiliary boiler. The profitability of CHPs system depends on the choice of the prime mover technology and size, and on the design of the combined TESS [32]. The high interdependence of the electrical and thermal energy generated by CHP can be modeled as reported below:

$$
\begin{aligned}
\mathbf{x}^{\operatorname{chp}}(k) & =\eta_{\mathrm{e}}^{\text {chp }} \mathbf{z}^{\operatorname{chp}}(k) \\
\mathbf{y}^{\operatorname{chp}}(k) & =\eta_{\mathrm{t}}^{\text {chp }} \mathbf{z}^{\text {chp }}(k) .
\end{aligned}
$$

The gas consumed by the CHP is represented by the input column vector $\mathbf{z}^{\text {chp }}(k)$, while the generated electrical and thermal energy are the output column vectors $\mathbf{x}^{\text {chp }}(k)$ and $\mathbf{y}^{\text {chp }}(k)$. Consequently the CHP presents both an electrical and a thermal efficiency respectively represented by $\eta_{\mathrm{e}}^{\mathrm{chp}}$ and $\eta_{\mathrm{t}}^{\mathrm{chp}}$, which are here assumed to be constant over the horizon [33], [34]. Operational requirements on the CHP unit impose to limit the generated electrical and thermal energy between minimum and maximum values $\underline{l}^{\text {chp }}, \bar{l}^{\text {chp }}, \underline{p}^{\text {chp }}$, and $\bar{p}^{\text {chp }}$ :

$$
\begin{aligned}
\underline{l}^{\mathrm{chp}} \mathbf{1}_{H} & \leq \mathbf{x}^{\mathrm{chp}}(k) \leq \bar{l}^{\mathrm{chp}} \mathbf{1}_{H} \\
\underline{p}^{\mathrm{chp}} \mathbf{1}_{H} & \leq \mathbf{y}^{\mathrm{chp}}(k) \leq \bar{p}^{\mathrm{chp}} \mathbf{1}_{H} .
\end{aligned}
$$

In addition, further constraints are imposed to limit the ramp up and ramp down for both the thermal and electrical generation of the CHP unit in accordance with the upward and downward ramp electrical rates $\underline{r}^{\text {chp }}$ and $\bar{r}^{\text {chp }}$ and thermal rates $\underline{\rho}^{\text {chp }}$ and $\bar{\rho}^{\text {chp }}$ :

$$
\begin{aligned}
& -\underline{r}^{\operatorname{chp}} \leq x^{\operatorname{chp}}(h)-x^{\operatorname{chp}}(h-1) \leq \bar{r}^{\mathrm{chp}}, \forall h \in[k+1, k+H] \\
& -\underline{\rho}^{\operatorname{chp}} \leq y^{\operatorname{chp}}(h)-y^{\operatorname{chp}}(h-1) \leq \bar{\rho}^{\mathrm{chp}}, \forall h \in[k+1, k+H] .
\end{aligned}
$$

\section{H. Electrical Energy Storage System}

The EESS can both provide and absorb electrical energy to and from the microgrid. Consequently, we consider two different vectors $\mathbf{x}_{-}^{\mathrm{es}}(k)$ and $\mathbf{x}_{+}^{\mathrm{es}}(k)$ to represent the discharging and charging activities of the EESS over the time horizon. The EESS is here represented as a first-order dynamic system whose charge level, given the charging and discharging efficiencies $\eta_{+}^{\mathrm{es}}$ and $\eta_{-}^{\mathrm{es}}$, can be modeled as follows:

$$
\begin{gathered}
s^{\mathrm{es}}(h)=s^{\mathrm{es}}(h-1)+\eta_{+}^{\mathrm{es}} x_{+}^{\mathrm{es}}(h)-\frac{1}{\eta_{-}^{\mathrm{es}}} x_{-}^{\mathrm{es}}(h), \\
\forall h \in[k+1, k+H] .
\end{gathered}
$$

The charge level is limited by the minimum and maximum EESS capacity $\underline{S}^{\mathrm{es}}$ and $\bar{S}^{\mathrm{es}}$ :

$$
\begin{gathered}
\underline{S}^{\mathrm{es}}-s^{\mathrm{es}}(h-1) \leq \eta_{+}^{\mathrm{es}} x_{+}^{\mathrm{es}}(h)-\frac{1}{\eta_{-}^{\mathrm{es}}} x_{-}^{\mathrm{es}}(h) \\
\leq \bar{S}^{\mathrm{es}}-s^{\mathrm{es}}(h-1), \forall h \in[k+1, k+H] .
\end{gathered}
$$

To represent the mutual exclusion of the charging/discharging events, i.e., the electrical energy can flow from the microgrid to the EESS and vice versa but not simultaneously, the model includes the following constraints:

$$
\begin{gathered}
\mathbf{0}_{H} \leq \mathbf{x}_{+}^{\mathrm{es}}(k) \leq \boldsymbol{\delta}_{+}^{\mathrm{es}}(k) \bar{s}^{\mathrm{es}} \\
\mathbf{0}_{H} \leq \mathbf{x}_{-}^{\mathrm{es}}(k) \leq \boldsymbol{\delta}_{-}^{\mathrm{es}}(k) \underline{s}^{\mathrm{es}} \\
\boldsymbol{\delta}_{+}^{\mathrm{es}}(k) \in\{0,1\}^{H}, \boldsymbol{\delta}_{-}^{\mathrm{es}}(k) \in\{0,1\}^{H} \\
\boldsymbol{\delta}_{+}^{\mathrm{es}}(k)+\boldsymbol{\delta}_{-}^{\mathrm{es}}(k) \leq \mathbf{1}_{H}
\end{gathered}
$$

where $\bar{s}^{\mathrm{es}}$ and $\underline{s}^{\mathrm{es}}$ are the maximum charging and discharging rates, while $\boldsymbol{\delta}_{+}^{\mathrm{es}}(k)$ and $\boldsymbol{\delta}_{-}^{\mathrm{es}}(k)$ are two supporting vectors over the time horizon $[k+1, k+H]$.

We highlight that $\delta_{+}^{\mathrm{es}}(h)=1$ or $\delta_{+}^{\mathrm{es}}(h)=0$ if the EESS is charged or is not charged at time step $h$, respectively; similarly, it holds $\delta_{-}^{\mathrm{es}}(h)=1$ or $\delta_{-}^{\mathrm{es}}(h)=0$ if the EESS is discharged or is not discharged at time step $h$, respectively.

\section{Thermal Energy Storage System}

The TESS is modeled similarly to the EESS, thus we consider vectors $\mathbf{y}_{+}^{\mathrm{ts}}(k)$ and $\mathbf{y}_{-}^{\mathrm{ts}}(k)$ respectively representing the decision variables related to the energy storage end provision. The charge level at time step $k$ is represented as $s^{\text {ts }}(k)$ and the constraints below are imposed:

$$
\begin{gathered}
s^{\mathrm{ts}}(h)=s^{\mathrm{ts}}(h-1)+\eta_{+}^{\mathrm{ts}} y_{+}^{\mathrm{ts}}(h)-\frac{1}{\eta_{-}^{\mathrm{ts}}} y_{-}^{\mathrm{ts}}(h), \\
\forall h \in[k+1, k+H] \\
\underline{S}^{\mathrm{ts}}-s^{\mathrm{ts}}(h-1) \leq \eta_{+}^{\mathrm{ts}} y_{+}^{\mathrm{ts}}(h)-\frac{1}{\eta_{-}^{\mathrm{ts}}} y_{-}^{\mathrm{ts}}(h) \\
\leq \bar{S}^{\mathrm{ts}}-s^{\mathrm{ts}}(h-1), \forall h \in[k+1, k+H] \\
\mathbf{0}_{H} \leq \mathbf{y}_{+}^{\mathrm{ts}}(k) \leq \boldsymbol{\delta}_{+}^{\mathrm{ts}}(k) \bar{s}^{\mathrm{ts}} \\
\mathbf{0}_{H} \leq \mathbf{y}_{-}^{\mathrm{ts}}(k) \leq \boldsymbol{\delta}_{-}^{\mathrm{ts}}(k) \underline{s}^{\mathrm{ts}} \\
\boldsymbol{\delta}_{+}^{\mathrm{ts}}(k) \in\{0,1\}^{H}, \boldsymbol{\delta}_{-}^{\mathrm{ts}}(k) \in\{0,1\}^{H} \\
\boldsymbol{\delta}_{+}^{\mathrm{ts}}(k)+\boldsymbol{\delta}_{-}^{\mathrm{ts}}(k) \leq \mathbf{1}_{H}
\end{gathered}
$$


where $\eta_{+}^{\mathrm{ts}}$ and $\eta_{-}^{\mathrm{ts}}$ are the charging and discharging efficiencies, $\underline{S}^{\text {ts }}$ and $\bar{S}^{\text {ts }}$ are the minimum and maximum capacities, $\bar{s}^{\text {ts }}$ and $\underline{s}^{\text {ts }}$ are the maximum charging and discharging rates, and $\boldsymbol{\delta}_{+}^{\mathrm{ts}}(k)$ and $\boldsymbol{\delta}_{-}^{\mathrm{ts}}(k)$ are two vectors of supporting variables with an analogous meaning as in the EESS case.

\section{J. Electrical and Thermal Demand-Supply Balance}

In presence of CHP units, microgrids deal also with the exchanged thermal energy flows. Hence, for the sake of satisfying the energy balance in the multi-carrier scenario, a demand-supply balance constraint must be imposed at any time slot for both the electrical and thermal energy flows.

Specifically, the local balance of the electrical energy flow over the time horizon is expressed through the following equation:

$$
\begin{aligned}
& \mathbf{x}^{\mathrm{gr}}(k)+\mathbf{x}^{\mathrm{chp}}(k)+\mathbf{x}_{-}^{\mathrm{es}}(k)+\mathbf{r}(k)= \\
& \sum_{n=1}^{N^{\mathrm{el}}} \mathbf{x}_{n}^{\mathrm{el}}(k)+\mathbf{x}^{\mathrm{hp}}(k)+\mathbf{x}_{+}^{\mathrm{es}}(k)+\mathbf{b}(k)
\end{aligned}
$$

where $\mathbf{x}^{\mathrm{gr}}(k)$ is a column vector denoting the profile of the electricity exchanged between the main grid and the microgrid and sold over the time horizon: for each at time step we have that $h \in[k+1, k+H] x^{\mathrm{gr}}(h) \geq 0$ if the microgrid buys energy from the grid, whilst $x^{\mathrm{gr}}(k)<0$ if the microgrid sells energy to the grid.

The balance equation (28) can be rewritten more compactly as follows:

$$
\mathbf{x}^{\mathrm{gr}}(k)=\mathbf{x}^{\mathrm{a}}(k)+\mathbf{d}(k)
$$

where we denote the net electrical demand as $\mathbf{d}(k):=\mathbf{b}(k)-$ $\mathbf{r}(k)$ and we introduce the supporting variables vector $\mathbf{x}^{\mathrm{a}}(k)$ defined as:

$$
\mathbf{x}^{\mathrm{a}}(k)=\sum_{n=1}^{N^{\mathrm{el}}} \mathbf{x}_{n}^{\mathrm{el}}(k)+\mathbf{x}^{\mathrm{hp}}(k)+\mathbf{x}_{+}^{\mathrm{es}}(k)-\mathbf{x}^{\mathrm{chp}}(k)-\mathbf{x}_{-}^{\mathrm{es}}(k) .
$$

Similarly, for the local balance of the thermal energy flow over the time horizon we have:

$$
\begin{gathered}
\mathbf{y}^{\mathrm{boi}}(k)+\mathbf{y}^{\mathrm{chp}}(k)+\mathbf{y}^{\mathrm{hp}}(k)+\mathbf{y}_{-}^{\mathrm{ts}}(k)= \\
\sum_{m=1}^{M^{\mathrm{ctl}}} \mathbf{y}_{m}^{\mathrm{ctl}}(k)+\mathbf{y}_{+}^{\mathrm{ts}}(k)+\mathbf{q}(k) .
\end{gathered}
$$

The balance equation (31) can be rewritten more compactly as follows:

$$
\mathbf{y}^{\mathrm{boi}}(k)=\mathbf{y}^{\mathrm{a}}(k)+\mathbf{q}(k)
$$

where we introduce the supporting variables vector $\mathbf{y}^{\mathrm{a}}(k)$ defined as:

$$
\mathbf{y}^{\mathrm{a}}(k)=\sum_{m=1}^{M^{\mathrm{ctl}}} \mathbf{y}_{m}^{\mathrm{ctl}}(k)+\mathbf{y}_{+}^{\mathrm{ts}}(k)-\mathbf{y}^{\operatorname{chp}}(k)-\mathbf{y}^{\mathrm{hp}}(k)-\mathbf{y}_{-}^{\mathrm{ts}}(k) .
$$

\section{K. Electricity and Gas Pricing and Contractual Constraints}

A contractual obligation restricts the energy that the microgrid can buy from and sell to the electricity provider at each time slot. Denoting the maximum purchasable and salable energy profile imposed by the power grid over the time horizon respectively as column vectors $\overline{\mathbf{e}}(k)$ and $\underline{\mathbf{e}}(k)$, the following constraints must be satified:

$$
\underline{\mathbf{e}}(k) \leq \mathbf{x}^{\mathrm{gr}}(k) \leq \overline{\mathbf{e}}(k) .
$$

The pricing function for the electricity exchanged with the power grid is assumed to be linear [21], [34] but different unit prices are considered. In particular, the buying and selling pricing coefficients over the time horizon are collected into two different vectors denotes as $\boldsymbol{\kappa}_{+}(k)$ and $\boldsymbol{\kappa}_{-}(k)$, respectively. The electricity cost incurred by the microgrid at time slot $h$ is thus defined as:

$$
\left\{\begin{array}{ll}
\kappa_{+}(h)^{\top} x^{\mathrm{gr}}(h), & \text { if } x^{\mathrm{gr}}(h) \geq 0 \\
\kappa_{-}(h)^{\top} x^{\mathrm{gr}}(h), & \text { if } x^{\mathrm{gr}}(h)<0
\end{array}, \forall h \in[k+1, k+H] .\right.
$$

Equation (35) can be transformed in a linear form using logic constraints as indicated in [35]. To this aim, denoting the vector collecting the logical variables over the time horizon as $\delta^{\mathrm{g}}(k)$, we introduce the following logical constraints:

$$
\mathbf{x}^{\mathrm{gr}}(k) \geq \mathbf{0}_{H} \Longleftrightarrow \boldsymbol{\delta}^{\mathrm{g}}(k)=\mathbf{0}_{H}
$$

and the following supporting variables:

$$
\mathbf{x}^{\mathrm{g} \delta}(k)=\boldsymbol{\delta}^{\mathrm{g}}(k) \circ \mathbf{x}^{\mathrm{gr}}(k) .
$$

Using the above defined supporting variables, (35) can be straightforwardly rewritten as follows:

$$
\begin{gathered}
c^{\mathrm{gr}}\left(\mathbf{x}^{\mathrm{gr}}(k), \mathbf{x}^{\mathrm{g} \delta}(k)\right)= \\
\boldsymbol{\kappa}_{+}(k)^{\top} \mathbf{x}^{\mathrm{gr}}(k)-\boldsymbol{\kappa}_{+}(k)^{\top} \mathbf{x}^{\mathrm{g} \delta}(k)+\boldsymbol{\kappa}_{-}(k)^{\top} \mathbf{x}^{\mathrm{g} \delta}(k) .
\end{gathered}
$$

Replacing (30) in (38) we get:

$$
\begin{gathered}
c^{\mathrm{gr}}\left(\mathbf{x}^{\mathrm{a}}(k), \mathbf{x}^{\mathrm{g} \delta}(k)\right)= \\
\boldsymbol{\kappa}_{+}(k)^{\top}\left(\mathbf{x}^{\mathrm{a}}(k)+\mathbf{d}(k)\right)-\boldsymbol{\kappa}_{+}(k)^{\top} \mathbf{x}^{\mathrm{g} \delta}(k)+\boldsymbol{\kappa}_{-}(k)^{\top} \mathbf{x}^{\mathrm{g} \delta}(k) .
\end{gathered}
$$

Following [35], (36) and (37) can be replaced with:

$$
\begin{gathered}
\mathbf{x}^{\mathrm{gr}}(k) \leq \overline{\mathbf{e}}(k) \circ\left(\mathbf{1}_{H}-\boldsymbol{\delta}^{\mathrm{g}}(k)\right) \\
\mathbf{x}^{\mathrm{gr}}(k) \geq \underline{\mathbf{e}}(k) \circ \boldsymbol{\delta}^{\mathrm{g}}(k) \\
\mathbf{x}^{\mathrm{g} \delta}(k) \leq \mathbf{x}^{\mathrm{gr}}(k)-\underline{\mathbf{e}}(k) \circ\left(\mathbf{1}_{H}-\boldsymbol{\delta}^{\mathrm{g}}(k)\right) \\
\mathbf{x}^{\mathrm{g} \delta}(k) \geq \mathbf{x}^{\mathrm{gr}}(k)-\overline{\mathbf{e}}(k) \circ\left(\mathbf{1}_{H}-\boldsymbol{\delta}^{\mathrm{g}}(k)\right) \\
\mathbf{x}^{\mathrm{g} \delta}(k) \leq \overline{\mathbf{e}}(k) \circ \boldsymbol{\delta}^{\mathrm{g}}(k) \\
\mathbf{x}^{\mathrm{g} \delta}(k) \geq \underline{\mathbf{e}}(k) \circ \boldsymbol{\delta}^{\mathrm{g}}(k) .
\end{gathered}
$$

Finally, the pricing function for the natural gas bought from the main grid is assumed to be linear [21], [34]. Denoting the vector of gas pricing coefficients over the time horizon as $\boldsymbol{\nu}(k)$, the cumulative gas cost incurred by the microgrid is thus:

$$
c^{\text {gas }}\left(\mathbf{z}^{\text {boi }}(k), \mathbf{z}^{\text {chp }}(k)\right)=\boldsymbol{\nu}(k)^{\top}\left(\mathbf{z}^{\text {boi }}(k)+\mathbf{z}^{\text {chp }}(k)\right) .
$$

A contractual obligation imposed by the gas provider restricts the quantity of natural gas that could be bought by the microgrid. Specifically, denoting the maximum purchasable 
gas amount over the time horizon as column vector $\overline{\mathbf{d}}(k)$, the following constraints must hold:

$$
\mathbf{0}_{H} \leq \mathbf{z}^{\text {boi }}(k)+\mathbf{z}^{\text {chp }}(k) \leq \overline{\mathbf{g}}(k) .
$$

\section{Deterministic Formulation of the Energy SCHEDULING PROBLEM}

In the deterministic approach, all the parameters are assumed unaffected by uncertainty. Specifically, the energy scheduling problem is formulated using the nominal forecasted values of electrical and thermal energy demand, RES generation, and electricity and natural gas coefficients.

The objective function aims at minimizing both the electricity and the gas cost defined in (39) and (46), respectively. Specifically, ignoring the constants, the sum of the terms in (39) and (46) can be compactly written as follows:

$$
\begin{gathered}
\boldsymbol{\kappa}_{+}(k)^{\top} \mathbf{x}^{\mathrm{a}}(k)+\left(\boldsymbol{\kappa}_{-}(k)-\boldsymbol{\kappa}_{+}(k)\right)^{\top} \mathbf{x}^{\mathrm{g} \delta}(k) \\
+\boldsymbol{\nu}(k)^{\top} \mathbf{z}^{\mathrm{boi}}(k)+\boldsymbol{\nu}(k)^{\top} \mathbf{z}^{\operatorname{chp}}(k)=\boldsymbol{\pi}(k)^{\top} \boldsymbol{\xi}(k)
\end{gathered}
$$

where we introduce the linear coefficients vector $\boldsymbol{\pi}(k):=$ $\left(\boldsymbol{\kappa}_{+}(k)^{\top},\left(\boldsymbol{\kappa}_{-}(k)-\boldsymbol{\kappa}_{+}(k)\right)^{\top}, \boldsymbol{\nu}(k)^{\top}, \boldsymbol{\nu}(k)^{\top}\right)^{\top}$ and the variables vector $\boldsymbol{\xi}(k):=\left(\mathbf{x}^{\mathrm{a}}(k)^{\top}, \mathbf{x}^{\mathrm{g} \delta}(k), \mathbf{z}^{\mathrm{boi}}(k)^{\top}, \mathbf{z}^{\mathrm{chp}}(k)^{\top}\right)^{\top}$.

The deterministic or nominal energy scheduling problem consists in determining the cost-optimal operation of the microgrid components over the time horizon $[k+1, k+H]$ such as the electricity consumption profile of CELs and HP, the amount of electricity to be bought from and sold to the power grid, the thermal energy consumption profile of TELs, the amount of natural gas to be bought for the CHP unit and the boiler, and the EESS and TESS charging/discharging strategy:

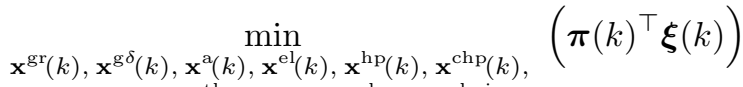

$$
\begin{aligned}
& \mathbf{x}_{+}^{\mathrm{es}}(k), \mathbf{x}_{-}^{\mathrm{es}}(k), \mathbf{y}^{\mathrm{ct}}(k), \mathbf{T}(k), \mathbf{y}^{\mathrm{chp}}(k), \mathbf{y}^{\mathrm{boi}}(k), \\
& \mathbf{y}^{\mathrm{a}}(k), \mathbf{y}_{+}^{\mathrm{ts}}(k), \mathbf{y}_{-}^{\mathrm{ts}}(k), \mathbf{z}^{\mathrm{boi}}(k), \mathbf{z}^{\mathrm{chp}}(k), \\
& \boldsymbol{\delta}_{+}^{\mathrm{es}}(k), \boldsymbol{\delta}_{-}^{\mathrm{es}}(k), \boldsymbol{\delta}_{+}^{\mathrm{ts}}(k), \boldsymbol{\delta}_{-}^{\mathrm{ts}}(k), \boldsymbol{\delta}^{\mathrm{g}}(k) \\
& \text { s.t. (1)-(27), (29)-(30), (32)-(34), (40)-(45), (47). }
\end{aligned}
$$

For the sake of simplifying (49) and omitting superfluous terms, we transform (49) as follows:

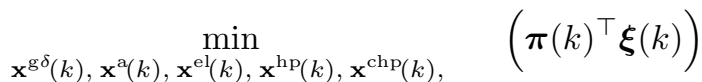

$$
\begin{aligned}
& \mathbf{x}_{+}^{\mathrm{es}}(k), \mathbf{x}_{-}^{\mathrm{es}}(k), \mathbf{y}^{\mathrm{ct}}(k), \mathbf{T}(k), \mathbf{y}^{\mathrm{chp}}(k), \mathbf{y}^{\mathrm{boi}}(k), \\
& \mathbf{y}_{(}^{\mathrm{a}}(k), \mathbf{y}_{+}^{\mathrm{ts}}(k), \mathbf{y}_{-}^{\mathrm{ts}}(k), \mathbf{z}^{\mathrm{boi}}(k), \mathbf{z}^{\mathrm{chp}}(k), \\
& \boldsymbol{\delta}_{+}^{\mathrm{es}}(k), \boldsymbol{\delta}_{-}^{\mathrm{es}}(k), \boldsymbol{\delta}_{+}^{\mathrm{ts}}(k), \boldsymbol{\delta}_{-}^{\mathrm{ts}}(k), \boldsymbol{\delta}^{\mathrm{g}}(k) \\
& \text { s.t. (1)-(27), (30), (32)-(33), and }
\end{aligned}
$$

$$
\begin{gathered}
\mathbf{x}^{\mathrm{a}}(k)+\mathbf{d}(k) \leq \overline{\mathbf{e}}(k) \circ\left(\mathbf{1}_{H}-\boldsymbol{\delta}^{\mathrm{g}}(k)\right) \\
\mathbf{x}^{\mathrm{a}}(k)+\mathbf{d}(k) \geq \underline{\mathbf{e}}(k) \circ \boldsymbol{\delta}^{\mathrm{g}}(k) \\
\mathbf{x}^{\mathrm{g} \delta}(k) \leq \mathbf{x}^{\mathrm{a}}(k)+\mathbf{d}(k)-\underline{\mathbf{e}}(k) \circ\left(\mathbf{1}_{H}-\boldsymbol{\delta}^{\mathrm{g}}(k)\right) \\
\mathbf{x}^{\mathrm{g} \delta}(k) \geq \mathbf{x}^{\mathrm{a}}(k)+\mathbf{d}(k)-\overline{\mathbf{e}}(k) \circ\left(\mathbf{1}_{H}-\boldsymbol{\delta}^{\mathrm{g}}(k)\right) \\
\underline{\mathbf{e}}(k) \leq \mathbf{x}^{\mathrm{a}}(k)+\mathbf{d}(k) \\
\mathbf{x}^{\mathrm{a}}(k)+\mathbf{d}(k) \leq \overline{\mathbf{e}}(k)
\end{gathered}
$$

$$
\begin{gathered}
\mathbf{y}^{\mathrm{a}}(k)+\eta^{\mathrm{boi}} \mathbf{z}^{\mathrm{chp}}(k)+\mathbf{q}(k) \leq \eta^{\mathrm{boi}} \overline{\mathbf{g}}(k) \\
\mathbf{y}^{\mathrm{a}}(k)+\eta^{\mathrm{boi}} \mathbf{z}^{\mathrm{chp}}(k)+\mathbf{q}(k) \geq \mathbf{0}_{H} .
\end{gathered}
$$

Note that equations (51)-(54) and (55)-(56) are obtained by replacing (29) in (40)-(43) and (34), respectively. Similarly, equations (57)-(58) are obtained replacing (32) and (11) in (47).

In particular, (50)-(58) is a mixed integer linear programming (MILP) problem that consists in determining $H(N+M+$ 14) real and $5 H$ binary decision variables, which minimize the objective function in (50), and meet $2 H(N+M+14)$ bounding constraints, $(H M+N+8 H)$ equality constraints, $24 H$ inequality constraints, and $H(N+2)$ integrality constraints.

The optimization problem (50)-(58) is iteratively solved at each time slot $k$ based on the most recent input data in accordance with the rolling horizon concept, thus defining the deterministic MPC approach. The results related to the first time slot are implemented in the microgrid as the optimal control signals. Subsequently, the horizon is shifted ahead at the next time slot: a new optimization problem is solved using the updated input data.

\section{Robust Formulation of THE ENERGy SCHEdULING PROBLEM}

The variation in the forecast of thermal energy demand, electrical demand, RES generation, and electricity and gas pricing coefficients (i.e., of vectors $\mathbf{q}, \mathbf{b}, \mathbf{b}, \boldsymbol{\kappa}_{+}, \boldsymbol{\kappa}_{-}$, and $\boldsymbol{\nu}$ ) may lead the deterministic scheduling to ineffective results, i.e., microgrid strategies that are suboptimal and even unfeasible. Differently from the previous deterministic scheduling approach, where unrealistically no perturbation is assumed to influence the nominal values, in this section we tackle uncertainty in the energy scheduling by defining a computationally tractable method able to determine robust solutions. In the first stage of the proposed method, a data set is selected within the uncertainty space, defining the so-called uncertainty set. In the second stage, the optimal solution that is feasible for any realization of uncertain parameters within the defined uncertainty set is computed. The optimization problem in the second stage is referred as robust counterpart optimization problem, whose complexity closely depends on the geometrical definition of the uncertainty set.

\section{A. Data Uncertainty Set}

We assume that the uncertain vectors of thermal energy demand, electrical demand, RES generation, and electricity and gas pricing coefficients - denoted as $\widetilde{\mathbf{q}}(k), \widetilde{\mathbf{b}}(k), \widetilde{\mathbf{r}}(k), \widetilde{\boldsymbol{\kappa}}_{+}(k)$, $\widetilde{\boldsymbol{\kappa}}_{-}(k)$, and $\widetilde{\boldsymbol{\nu}}(k)$, respectively - are modeled as follows:

$$
\begin{aligned}
& \mathbf{q}(k)-\hat{\mathbf{q}}(k) \leq \widetilde{\mathbf{q}}(k) \leq \mathbf{q}(k)+\hat{\mathbf{q}}(k) \\
& \mathbf{b}(k)-\hat{\mathbf{b}}(k) \leq \widetilde{\mathbf{b}}(k) \leq \mathbf{b}(k)+\hat{\mathbf{b}}(k) \\
& \mathbf{r}(k)-\hat{\mathbf{r}}(k) \leq \widetilde{\mathbf{r}}(k) \leq \mathbf{r}(k)+\hat{\mathbf{r}}(k) \\
& \boldsymbol{\kappa}_{+}(k)-\hat{\boldsymbol{\kappa}}_{+}(k) \leq \widetilde{\boldsymbol{\kappa}}_{+}(k) \leq \boldsymbol{\kappa}_{+}(k)+\hat{\boldsymbol{\kappa}}_{+}(k) \\
& \boldsymbol{\kappa}_{-}(k)-\hat{\boldsymbol{\kappa}}_{-}(k) \leq \widetilde{\boldsymbol{\kappa}}_{-}(k) \leq \boldsymbol{\kappa}_{-}(k)+\hat{\boldsymbol{\kappa}}_{-}(k) \\
& \boldsymbol{\nu}(k)-\hat{\boldsymbol{\nu}}(k) \leq \widetilde{\boldsymbol{\nu}}(k) \leq \boldsymbol{\nu}(k)+\hat{\boldsymbol{\nu}}(k)
\end{aligned}
$$


where $\hat{\mathbf{q}}(k), \hat{\mathbf{b}}(k), \hat{\mathbf{r}}(k), \hat{\boldsymbol{\kappa}}_{+}(k), \hat{\boldsymbol{\kappa}}_{-}(k), \hat{\boldsymbol{\nu}}(k)$ are the vectors collecting the semi-amplitude of maximum variations of thermal energy demand, net electrical demand, and pricing coefficients, respectively. Note that both the nominal and semiamplitude values (i.e., vectors marked with tilde $\sim$ and hat $\wedge$, respectively) are computed by forecast algorithms that are assumed available as well as historical data. Consequently, the uncertainty model (59)-(64) is defined using a variation interval centered on the nominal profile rather than identifying the probability distribution of the uncertain parameters.

The above described sources of uncertainty affect the linear coefficients $\pi$ of the objective function (48), the linear coefficients $\mathbf{d}$ of constraints (51)-(56), and the linear coefficients of constraints (57)-(58). Note that the distribution of value for parameters vectors $\widetilde{\mathbf{d}}(k)$ and $\widetilde{\boldsymbol{\pi}}(k)$ is derived from the uncertainty space defined by (60)-(61) and (62)-(64), respectively:

$$
\begin{aligned}
\mathbf{d}(k)-\hat{\mathbf{d}}(k) & \leq \widetilde{\mathbf{d}}(k) \leq \mathbf{d}(k)+\hat{\mathbf{d}}(k) \\
\boldsymbol{\pi}(k)-\hat{\boldsymbol{\pi}}(k) & \leq \widetilde{\boldsymbol{\pi}}(k) \leq \boldsymbol{\pi}(k)+\hat{\boldsymbol{\pi}}(k)
\end{aligned}
$$

where $\hat{\mathbf{d}}(k) \quad:=\hat{\mathbf{b}}(k)+\hat{\mathbf{r}}(k)$ and $\hat{\boldsymbol{\pi}}(k) \quad:=$ $\left(\hat{\boldsymbol{\kappa}}_{+}(k)^{\top},\left(\hat{\boldsymbol{\kappa}}_{-}(k)+\hat{\boldsymbol{\kappa}}_{+}(k)\right)^{\top}, \hat{\boldsymbol{\nu}}(k)^{\top}, \hat{\boldsymbol{\nu}}(k)^{\top}\right)^{\top}$.

Rather than adopting the worst case protection against the maximum deviation of uncertainty parameters (i.e., $\widetilde{\mathbf{q}}(k)$, $\widetilde{\mathbf{d}}(k)$, and $\widetilde{\boldsymbol{\pi}}(k)$ ) [36], several approaches have been proposed in the literature to deal with uncertainty minimizing the impact on objective function and constraints [37]. Differently from [20], where an adjustable box-uncertainty set method is considered, we follow the cardinality-constrained approach proposed in [28]. To this aim, we introduce the robustness factors (also known as budgets of uncertainty) $\gamma_{q}, \gamma_{d}$, and $\gamma_{\pi}$ related respectively to the thermal energy demand, the net electrical demand, and pricing coefficients, to control the degree of conservatism of the solution.

As for $\gamma_{q}$, this robustness factor takes values in $[0, H]$ indicating the number of parameters (i.e., $q(h), h \in[k+1, k+H]$ ) protected against disturbances. The energy scheduling solution is ensured to be feasible if no more than $\left\lfloor\gamma_{q}\right\rfloor$ of the elements in $\widetilde{\mathbf{q}}(k)$ are subject to uncertainty, and one of them $\widetilde{q}(h)$ changes no more than $\left(\gamma_{q}-\left\lfloor\gamma_{q}\right\rfloor\right) \hat{q}(h)$. Analogous meaning holds for $\gamma_{d}$, taking values in $[0, H]$ as well; finally, for $\gamma_{\pi}$ the meaning is similar except for the range that takes value in $[0,4 H]$.

\section{B. Robust MPC Approach}

Substituting vectors of nominal profiles $\mathbf{q}(k), \mathbf{d}(k), \boldsymbol{\pi}(k)$ with vectors affected by uncertainty $\widetilde{\mathbf{q}}(k), \widetilde{\mathbf{d}}(k)$, and $\widetilde{\boldsymbol{\pi}}(k)-$ which take values in the uncertainty space defined by (59), (65), and (66)- the energy scheduling problem (50)-(58) is transformed into a robust optimization problem. Following [28], having defined the robustness factors $\gamma_{q}, \gamma_{d}$, and $\gamma_{\pi}$, we now formulate the robust counterpart optimization model as follows:

$$
\begin{aligned}
& \min _{\mathbf{x}^{\mathrm{g} \delta}(k), \mathbf{x}^{\mathrm{a}}(k), \mathbf{x}^{\mathrm{el}}(k), \mathbf{x}^{\mathrm{hp}}(k),}\left(\boldsymbol{\pi}(k)^{\top} \boldsymbol{\xi}(k)+\beta\left(\boldsymbol{\xi}(k), \gamma_{\pi}\right)\right) \\
& \mathbf{x}^{\mathrm{chp}}(k), \mathbf{x}_{+}^{\mathrm{es}}(k), \mathbf{x}_{-}^{\mathrm{es}}(k), \mathbf{y}^{\mathrm{ctl}}(k), \\
& \mathbf{T}(k), \mathbf{y}^{\mathrm{chp}}(k), \mathbf{y}^{\mathrm{boi}}(k), \mathbf{y}^{\mathrm{a}}(k), \\
& \mathbf{y}_{+}^{\mathrm{ts}}(k), \mathbf{y}_{-}^{\mathrm{ts}}(k), \mathbf{z}^{\mathrm{boi}}(k), \mathbf{z}^{\mathrm{chp}}(k), \\
& \boldsymbol{\delta}_{+}^{\mathrm{es}}(k), \boldsymbol{\delta}_{-}^{\mathrm{es}}(k), \boldsymbol{\delta}_{+}^{\mathrm{ts}}(k), \boldsymbol{\delta}_{-}^{\mathrm{ts}}(k), \boldsymbol{\delta}^{\mathrm{g}}(k)
\end{aligned}
$$

s.t. (1)-(27), (30), (32)-(33), and

$$
\begin{gathered}
\mathbf{x}^{\mathrm{a}}(k)+\overline{\mathbf{e}}(k) \circ \boldsymbol{\delta}^{\mathrm{g}}(k)+\mathbf{d}(k)+\boldsymbol{\psi}\left(\gamma_{d}\right) \leq \overline{\mathbf{e}}(k) \\
\mathbf{x}^{\mathrm{a}}(k)-\mathbf{e}(k) \circ \boldsymbol{\delta}^{\mathrm{g}}(k)+\mathbf{d}(k)-\boldsymbol{\psi}\left(\gamma_{d}\right) \geq \mathbf{0}_{H} \\
\mathbf{x}^{\mathrm{a}}(k)-\mathbf{x}^{\mathrm{g} \delta}(k)+\overline{\mathbf{e}}(k) \circ \boldsymbol{\delta}^{\mathrm{g}}(k)+\mathbf{d}(k)-\boldsymbol{\psi}\left(\gamma_{d}\right) \geq \overline{\mathbf{e}}(k) \\
\mathbf{x}^{\mathrm{a}}(k)-\mathbf{x}^{\mathrm{g} \delta}(k)+\overline{\mathbf{e}}(k) \circ \boldsymbol{\delta}^{\mathrm{g}}(k)+\mathbf{d}(k)+\boldsymbol{\psi}\left(\gamma_{d}\right) \leq \overline{\mathbf{e}}(k) \\
\mathbf{x}^{\mathrm{a}}(k)+\mathbf{d}(k)-\boldsymbol{\psi}\left(\gamma_{d}\right) \geq \mathbf{e}(k) \\
\mathbf{x}^{\mathrm{a}}(k)+\mathbf{d}(k)+\boldsymbol{\psi}\left(\gamma_{d}\right) \leq \overline{\mathbf{e}}(k) \\
\mathbf{y}^{\mathrm{a}}(k)+\eta^{\mathrm{boi}} \mathbf{z}^{\operatorname{chp}}(k)+\mathbf{q}(k)+\boldsymbol{\varphi}\left(\gamma_{q}\right) \leq \eta^{\mathrm{boi}} \mathbf{g}(k) \\
\mathbf{y}^{\mathrm{a}}(k)+\eta^{\mathrm{boi}} \mathbf{z}^{\operatorname{chp}}(k)+\mathbf{q}(k)-\boldsymbol{\varphi}\left(\gamma_{q}\right) \geq \mathbf{0}_{H}
\end{gathered}
$$

where:

$$
\begin{aligned}
& \beta\left(\boldsymbol{\xi}(k), \gamma_{\pi}\right)= \\
& \max _{\substack{\left\{\mathcal{V} \cup\{v\} \\
\mathcal{V} \mid=\left\lfloor\gamma_{\pi}\right\rfloor, v \in \mathbb{N}_{[0,4 H]} \backslash \mathcal{V}\right\}}}\left(\sum_{h \in \mathbb{N}_{[0,4 H]}} \hat{\pi}(h)|\xi(h)|\right. \\
& \left.+\left(\gamma_{\pi}-\left\lfloor\gamma_{\pi}\right\rfloor\right) \hat{\pi}(v)|\xi(v)|\right) \\
& \boldsymbol{\psi}\left(\gamma_{d}\right)=\left[\begin{array}{c}
\psi_{1}\left(\gamma_{d}\right) \\
\vdots \\
\psi_{H}\left(\gamma_{d}\right)
\end{array}\right]=\max _{u(1), \ldots, u(H)}\left[\begin{array}{c}
u(1) \hat{d}(1) \\
\vdots \\
u(H) \hat{d}(H)
\end{array}\right] \\
& \text { s.t. } \quad 0 \leq u(h) \leq 1, h \in[k+1, k+H] \\
& \sum_{h=\in[k+1, k+H]} u(h) \leq \gamma_{d} \\
& \boldsymbol{\varphi}\left(\gamma_{q}\right)=\left[\begin{array}{c}
\varphi_{1}\left(\gamma_{q}\right) \\
\vdots \\
\varphi_{H}\left(\gamma_{q}\right)
\end{array}\right]=\max _{u(1), \ldots, u(H)}\left[\begin{array}{c}
u(1) \hat{q}(1) \\
\vdots \\
u(H) \hat{q}(H)
\end{array}\right] \\
& \text { s.t. } 0 \leq u(h) \leq 1, h \in[k+1, k+H] \\
& \sum_{h \in[k+1, k+H]} u(h) \leq \gamma_{q} .
\end{aligned}
$$

We preliminarily note that (67)-(75) is a nonlinear optimization problem due to the nonlinearities introduced by the function $\beta\left(\boldsymbol{\xi}(k), \gamma_{\pi}\right)$ (denoted as the protection function for the objective) and the functions $\boldsymbol{\psi}\left(\gamma_{q}\right)$ and $\boldsymbol{\varphi}\left(\gamma_{q}\right)$ (each denoted as the protection function for the inequality constraints). As for the protection function $\beta\left(\boldsymbol{\xi}(k), \gamma_{\pi}\right)$, in (76) we introduce the subset $\mathcal{V}$ and the index $v$ to deal with the cardinalityconstrained uncertainty. In particular, $\mathcal{V}$ is the subset of time slot indices whose corresponding cost coefficients get the maximum deviation from the nominal values. At most $\left\lfloor\gamma_{\pi}\right\rfloor$ indices are assumed to belong to this subset. Further, in case $\gamma_{\pi}$ is not integer, we select a time slot index $v$, whose corresponding cost coefficient is affected by a variation lower than the maximum deviation (i.e., the value is between $\pi(v)$ and $\pi(v)+\hat{\pi}(v)$ ). All the remaining cost coefficient get the nominal values (i.e., $\pi(h)$ for $h$ not belonging to $\mathcal{V}$ and different from $v$ ). Similarly, both in (77)-(79) and (77)-(79), we introduce the $H$ decision variables $u(1), \ldots, u(H)$ to quantify 
the portions (not necessarily integer) of the total uncertainty budgets $\gamma_{d}$ and $\gamma_{q}$ allocated over all the time slots.

Moreover, we remark that tuning the values of $\gamma_{q}, \gamma_{d}$, and $\gamma_{\pi}$ different robustness levels can be allocated to the achievable energy scheduling strategies. On the one hand, for $\gamma_{b}=\gamma_{q}=\gamma_{r}=0$ no robustness is required to the problem resolution: this corresponds to disregard the forecast uncertainty and refer to the deterministic scheduling, thus addressing the most optimistic case. On the other hand, for $\gamma_{q}=\gamma_{q}=H$ and $\gamma_{\pi}=4 H$ the highest robustness is required to the problem resolution: this means that the largest variation of parameters is considered, thus addressing the most conservative case. For the sake of achieving a tradeoff between the level of conservativeness and the cost of the solution, the budgets of uncertainty $\gamma_{q}, \gamma_{d}$, and $\gamma_{\pi}$ can be tuned between intermediate value in the range $[0, H]$ and $[0,4 H]$, respectively [38].

We finally note that, likewise to the deterministic approach in (49), the robust problem (67)-(75) is solved iteratively at each time slot $k$ following the rolling horizon principle: the corresponding closed-loop control algorithm is denoted as robust MPC (RMPC).

\section{Reformulation of the Robust MPC Approach}

The robust counterpart of the energy scheduling problem in its min-max formulation (67)-(75) is hard to solve due to the presence of the inner maximization problems and the cardinality constraints included in the protection functions. Hence, we now present the derivation procedure of a tractable equivalent robust counterpart optimization model.

We preliminarily introduce the supporting time-varying variables $\mu(k) \in \mathbb{R}, \boldsymbol{\theta}(k) \in \mathbb{R}^{4 H}, \boldsymbol{\zeta}(k) \in \mathbb{R}^{H}$, and $\boldsymbol{v}(k) \in \mathbb{R}^{H}$. Getting inspiration from [28] and using the duality theory, it can be demonstrated that the robust counterpart (67)-(75) is equivalent to the following MILP formulation:

$$
\begin{aligned}
& \min _{\mathbf{x}_{\mathrm{g} \delta}(k), \mathbf{x}^{\mathrm{a}}(k), \mathbf{x}^{\mathrm{el}}(k), \mathbf{x}^{\mathrm{hp}}(k),}\left(\boldsymbol{\pi}(k)^{\top} \boldsymbol{\xi}(k)+\gamma_{\pi} \mu(k)+\mathbf{1}_{4 H}^{\top} \boldsymbol{\theta}(k)\right) \\
& \mathbf{x}^{\mathrm{chp}}(k), \mathbf{x}_{+}^{\mathrm{es}}(k), \mathbf{x}_{-}^{\mathrm{es}}(k), \mathbf{y}^{\mathrm{ctl}}(k), \\
& \mathbf{T}(k), \mathbf{y}^{\mathrm{chp}}(k), \mathbf{y}^{\mathrm{bo}}(k), \mathbf{y}^{\mathrm{a}}(k), \\
& \mathbf{y}_{+}^{\mathrm{ts}}(k), \mathbf{y}_{-}^{\mathrm{ts}}(k), \mathbf{z}^{\mathrm{boi}}(k), \mathbf{z}^{\mathrm{chp}}(k), \\
& \boldsymbol{\delta}_{+}^{\mathrm{es}}(k), \boldsymbol{\delta}_{-}^{\mathrm{es}}(k), \boldsymbol{\delta}_{+}^{\mathrm{ts}}(k), \boldsymbol{\delta}_{-}^{\mathrm{ts}}(k), \boldsymbol{\delta}^{\mathrm{g}}(k) \\
& \mu(k), \boldsymbol{\theta}(k), \boldsymbol{\zeta}(k), \boldsymbol{v}(k)
\end{aligned}
$$

$$
\begin{gathered}
\text { s.t. (1)-(27), (30), (32)-(33), and } \\
\hat{\boldsymbol{\pi}}(k) \circ \boldsymbol{\xi}(k)-\mu(k) \mathbf{1}_{4 H}-\boldsymbol{\theta}(k) \leq \mathbf{0}_{4 H} \\
\mathbf{x}^{\mathrm{a}}(k)+\overline{\mathbf{e}}(k) \circ \boldsymbol{\delta}^{\mathrm{g}}(k)+\hat{\mathbf{d}} \circ \boldsymbol{\zeta}(k) \leq \overline{\mathbf{e}}(k)-\mathbf{d}(k) \\
\mathbf{x}^{\mathrm{a}}(k)-\underline{\mathbf{e}}(k) \circ \boldsymbol{\delta}^{\mathrm{g}}(k)-\hat{\mathbf{d}} \circ \boldsymbol{\zeta}(k) \geq-\mathbf{d}(k) \\
\mathbf{x}^{\mathrm{a}}(k)-\mathbf{x}^{\mathrm{g} \delta}(k)+\overline{\mathbf{e}}(k) \circ \boldsymbol{\delta}^{\mathrm{g}}(k)-\hat{\mathbf{d}} \circ \boldsymbol{\zeta}(k) \geq \overline{\mathbf{e}}(k)-\mathbf{d}(k)(87) \\
\mathbf{x}^{\mathrm{a}}(k)-\mathbf{x}^{\mathrm{g} \delta}(k)+\overline{\mathbf{e}}(k) \circ \boldsymbol{\delta}^{\mathrm{g}}(k)+\hat{\mathbf{d}} \circ \boldsymbol{\zeta}(k) \leq \overline{\mathbf{e}}(k)-\mathbf{d}(k)(88) \\
\mathbf{x}^{\mathrm{a}}(k)+\mathbf{d}(k)-\hat{\mathbf{d}} \circ \boldsymbol{\zeta}(k) \geq \underline{\mathbf{e}}(k) \\
\mathbf{x}^{\mathrm{a}}(k)+\mathbf{d}(k)+\hat{\mathbf{d}} \circ \boldsymbol{\zeta}(k) \leq \overline{\mathbf{e}}(k) \\
\mathbf{y}^{\mathrm{a}}(k)+\eta^{\mathrm{boi}} \mathbf{z}^{\operatorname{chp}}(k)+\hat{\mathbf{q}} \circ \boldsymbol{v}(k) \\
\leq \eta^{\mathrm{boi}} \overline{\mathbf{g}}(k)-\mathbf{q}(k) \\
\mathbf{y}^{\mathrm{a}}(k)+\eta^{\mathrm{boi}} \mathbf{z}^{\operatorname{chp}}(k)-\hat{\mathbf{q}} \circ \boldsymbol{v}(k) \geq-\mathbf{q}(k)
\end{gathered}
$$

$$
\begin{gathered}
\mu(k) \geq 0 \\
\boldsymbol{\theta}(k) \geq \mathbf{0}_{4 H} \\
\mathbf{0}_{H} \leq \boldsymbol{\zeta}(k) \leq \mathbf{1}_{H} \\
\mathbf{1}_{H}^{\top} \boldsymbol{\zeta}(k) \geq \gamma_{d} \\
\mathbf{0}_{H} \leq \boldsymbol{v}(k) \leq \mathbf{1}_{H} \\
\mathbf{1}_{H}^{\top} \boldsymbol{v}(k) \geq \gamma_{q} .
\end{gathered}
$$

Finally, we remark that (83)-(98) is an MILP optimization problem that consists in determining $H(N+M+20)+1$ real and $5 H$ binary decision variables, which minimize the objective function in (83), and meet $2(H(N+M+20)+1)$ bounding constraints, $(H M+N+8 H)$ equality constraints, $30 H$ inequality constraints, and $H(N+2)$ integrality constraints.

As a final remark, we note that the proposed RMPC approach based on (83)-(98) shows several advantages with respect to other related methods. First, the approach relies on the uncertainty data model (59)-(64), which makes use only of the nominal and the variation profiles related to the uncertain parameters. Hence, differently from stochastic approaches which typically consider random variables and requires detailed statistical information on such variables, here uncertainty modeling is based on a small amount of information on the disturbances. Second, the reformulated optimization problem (83)-(98) is a computationally tractable program, which is not the typical case for other robust approach such as the stochastic [13] or the multi-stage robust optimization approach [39]. Lastly, by suitably setting the values of the budgets of uncertainty, the conservativeness of the RMPC solution can be directly controlled.

\section{NUMERICAL EXPERIMENTS}

In this section we apply the proposed robust control scheme to the online scheduling of a realistic residential multi-carrier microgrid. Considering that the system grid operators generally impose by contract to plan one day in advance the nominal value of energy exchange between the microgrid and the main grid and that the load and the renewable generation patterns are cyclic with a 24 hours time period (see, e.g., [14], [40], [41]), the prediction horizon is set to one day, whilst the sampling time is set to 1 hour (i.e., $H=24, \Delta \mathrm{h}=1$ hour). The effectiveness of the proposed method is tested over a simulation horizon $[1, T]$ of one year (i.e., $T=8760$ ) using the following four microgrid performance indices:

- the overall energy cost (EC) - taking into account both the gas and electricity costs incurred by the microgrid, it is calculated as the sum of $c^{\text {gr }}$ and $c^{\text {gas }}$ over the yearly time horizon, as:

$$
\begin{gathered}
\mathrm{EC}= \\
\sum_{k=1}^{T}\left(\kappa_{+}(k) x_{+}^{\mathrm{gr}}(k)-\kappa_{-}(k) x_{-}^{\mathrm{gr}}(k)+\nu(k)\left(z^{\mathrm{boi}}(k)+z^{\mathrm{chp}}(k)\right)\right) .
\end{gathered}
$$

- the self-supply (SS) - taking values in the interval $[0,1]$, it is calculated as the difference between 1 and the ratio 
between the energy sold to the grid and the energy generated in the microgrid by the RES and CHP:

$$
\mathrm{SS}=1-\frac{\sum_{k=1}^{T} x_{-}^{\mathrm{gr}}(k)}{\sum_{k=1}^{T} r(k)+x^{\operatorname{chp}(k)}} .
$$

- the fuel energy saving ratio (FESR) - taking values in the interval $[0,1]$, it is calculated as the difference between 1 and the ratio between the thermal energy produced by CHP and boiler and the thermal demand:

$$
\mathrm{FESR}=1-\frac{\sum_{k=1}^{T} x^{\mathrm{boi}}(k)+x^{\mathrm{chp}}(k)}{\sum_{k=1}^{T} q(k)+\sum_{m=1}^{M^{\mathrm{ctl}}} y_{m}^{\mathrm{ctl}}(k)} .
$$

- the energy independence (EI) - taking values in the interval $[0,1]$, it is calculated as the difference between 1 and the ratio between the energy bought from the grid and the energy consumed by CELs, NCELs, and HP:

$$
\mathrm{EI}=1-\frac{\sum_{k=1}^{T} x_{+}^{\mathrm{gr}}(k)}{\sum_{k=1}^{T} x^{\mathrm{hp}}(k)+\sum_{n=1}^{N^{\mathrm{el}}} x_{n}^{\mathrm{el}}(k)+b(k)} .
$$

In addition, for the sake of evaluating the robustness of the achieved strategies, the following two well-known robust optimization indices are employed:

- the price of robustness (PoR) - It is defined as the percentage of relative difference between the energy costs obtained by a robust solution and a nominal one.

- the constraint violation rate (CVR) - It is computed as the percentage of times a given solution does not satisfy the constraints affected by uncertainty over a given number of realizations of the uncertain parameters (e.g., through the runs of a Monte Carlo (MC) simulation).

Note that the PoR measures the optimality deviation of the robust solution with respect to the nominal one, whilst the CVR measures the robustness of the robust solution with respect to the worst case.

\section{A. System Parameters and Settings}

The considered multi-carrier microgrid system is installed in a residential district in the Netherlands, where $N=10$ households equipped with electrical and thermal loads (NCELs, CELs, NCTLs, and CTLs) share photovoltaic (PV) panels, a HP, a CHP unit, an EESS, and a TESS, as specified in Section II and depicted in Fig. 1.

As for the electrical and thermal demand, the corresponding profiles are excerpted from the aggregated Dutch national consumption curves [42] for year 2018. In particular, for each household an amount of 3.5 MWh and 14.0 MWh is considered on average as the yearly demand of electricity and thermal energy, respectively.

The total household electricity consumption is divided between NCELs (2 MWh) and CELs (1.5 MWh). Denoting the hourly residential electrical demand profile in [42] as $B(k)$ [kWh] and the hourly average residential electrical consumption over one year as $D_{\mathrm{Y}}^{\mathrm{el}}[\mathrm{kWh}]$, the hourly profile $b(k)[\mathrm{kWh}]$ of the NCELs is thus determined as follows:

$$
b(k)=N \frac{B(k)}{\sum_{i=1}^{T} B(i)} D_{\mathrm{Y}}^{\mathrm{el}}, k \in[1, T] .
$$

The corresponding semi-amplitude of maximum variations (i.e., $\hat{b}(k)$ ) of the electrical energy demand is determined as $15 \%$ of the nominal value $b(k)$ for each time slot.

In addition to the NCELs, the microgrids comprehends $N^{\text {cel }}=10$ CELs that are characterized by the following parameters whose values are reported in Table I: the cumulative daily energy consumption $L_{1}^{\mathrm{el}}, \ldots, L_{10}^{\mathrm{el}}$; the lower and upper bounds for the hourly consumption profiles $\underline{l}_{1}^{\mathrm{el}}, \ldots, \underline{l}_{10}^{\mathrm{el}}$ and $\bar{l}_{1}^{\mathrm{el}}, \ldots, \bar{l}_{10}^{\mathrm{el}}$.

Similarly to the electrical demand, the total household electricity consumption is divided between NCTLs (4 MWh) and CTLs (10 MWh). Denoting the hourly residential thermal demand profile in [42] as $Q(k)$ [kWh] and the hourly average residential electrical consumption over one year as $D_{\mathrm{Y}}^{\mathrm{tl}}[\mathrm{kWh}]$, the hourly profile $q(k)$ [kWh] of the NCTLs is thus determined as follows:

$$
q(k)=N \frac{Q(k)}{\sum_{i=1}^{T} Q(i)} D_{\mathrm{Y}}^{\mathrm{tl}}, k \in[1, T] .
$$

The corresponding semi-amplitude of maximum variations (i.e., $\hat{q}(k)$ ) of the thermal energy demand is determined as $15 \%$ of the nominal value $q(k)$ for each time slot.

In addition to the NCTLs, the microgrids comprehends $M^{\text {ctl }}=10$ CTLs that are characterized by the following parameters: the settling time of the indoor environment $(\tau)$ is set to $1.5 \mathrm{~h}$; the occupants impose the thermal comfort in the range $[19-22]{ }^{\circ} \mathrm{C}$ from $17 \mathrm{pm}$ to $8 \mathrm{am}$. The external temperature is based on hourly averaged measurements [42].

As for the energy generation, we consider the following types of technologies in the microgrid: PV panel, HP, CHP unit, and auxiliary boiler. All the technical parameters (such as operational boundaries, efficiency, etc.) that characterize these components are reported in Table I in terms of values and units. In particular, the PV panel has $30 \mathrm{kWp}$ capacity, whilst the production curve is based on the 2018 Dutch solar power time series [43].

Furthermore, we assume that the microgrid is equipped with storage technologies. Specifically, the EESS and TESS are represented by a lithium-ion battery and a water storage system for water and space heating, respectively. All the technical storage parameters (such as efficiency, capacity etc.) are reported in Table I in terms of values and units.

Finally, the prices of the energy and natural gas are set considering the average price in the Netherlands. The price of electricity is based on the spot price on the Nord Pool electricity market [44]. The buying price (i.e., $\kappa_{+}(k)$ ) is determined adding to the Nord Pool spot price fees (equal to $0.03 € / \mathrm{kWh}$ ), while selling price (i.e., $\kappa_{-}(k)$ ) is the spot price. As for the energy exchange of the microgrid with the distribution grid, we set a maximum hourly buying energy quantity $\bar{e}(t)=16 \mathrm{kWh}$ and a maximum hourly selling energy quantity $\underline{e}(t)=8 \mathrm{kWh}$. Conversely, the natural gas price is considered constant and equals $\nu(k)=0.08 € / \mathrm{kWh}$ [45], while the maximum hourly buying gas quantity is set to $\bar{g}(t)=40 \mathrm{~m}^{3}$.

Finally, similarly to the electrical and thermal energy demand, the corresponding semi-amplitude of maximum variations of the electricity and gas pricing (i.e., $\hat{\kappa_{+}}(k), \hat{\kappa_{-}}(k)$, 
TABLE I

TECHNICAL PARAMETERS OF THE MICROGRID COMPONENTS.

\begin{tabular}{|c|c|c|}
\hline Parameter & Value & Unit \\
\hline$\eta_{\mathrm{e}}^{\text {chp }}$ & 0.20 & - \\
\hline$\eta_{\mathrm{t}}^{\text {chp }}$ & 0.80 & - \\
\hline$\underline{l}^{\text {chp }}$ & 1 & $\mathrm{kWh}$ \\
\hline $\bar{l}^{\text {chp }}$ & 14 & $\mathrm{kWh}$ \\
\hline$\eta^{\text {boi }}$ & 1 & - \\
\hline$\underline{p}^{\text {boi }}$ & 1 & $\mathrm{kWh}$ \\
\hline $\bar{p}^{\text {boi }}$ & 15 & $\mathrm{kWh}$ \\
\hline$\underline{p}^{\text {chp }}$ & 8 & $\mathrm{kWh}$ \\
\hline $\bar{p}^{\text {chp }}$ & 56 & $\mathrm{kWh}$ \\
\hline$\eta^{\mathrm{hp}}$ & 3.5 & - \\
\hline$\underline{p}^{\text {hp }}$ & 3.5 & $\mathrm{kWh}$ \\
\hline $\bar{p}^{\mathrm{hp}}$ & 42 & $\mathrm{kWh}$ \\
\hline$\underline{r}^{\text {chp }}, \bar{r}^{\text {chp }}$ & 10 & $\mathrm{kWh}$ \\
\hline$\underline{\rho}^{\text {chp }}, \bar{\rho}^{\text {chp }}$ & 10 & $\mathrm{kWh}$ \\
\hline$\underline{\rho}^{\mathrm{hp}}, \bar{\rho}^{\mathrm{hp}}$ & 8 & $\mathrm{kWh}$ \\
\hline$\eta_{+}^{\mathrm{es}}, \eta_{-}^{\mathrm{es}}$ & 0.95 & - \\
\hline$\eta_{+}^{\mathrm{ts}}, \eta_{-}^{\mathrm{ts}}$ & 0.95 & - \\
\hline$\underline{s}^{\mathrm{es}}, \underline{s}^{\mathrm{ts}}$ & 0 & $\mathrm{kWh}$ \\
\hline $\bar{s}^{\mathrm{es}}$ & 10 & $\mathrm{kWh}$ \\
\hline $\bar{s}^{\mathrm{ts}}$ & 8 & $\mathrm{kWh}$ \\
\hline$\underline{S}^{\mathrm{es}}, \underline{S}^{\mathrm{ts}}$ & 0 & $\mathrm{~kW}$ \\
\hline
\end{tabular}

\begin{tabular}{|c|c|c|}
\hline Parameter & Value & Unit \\
\hline $\bar{S}^{\mathrm{es}}$ & 40 & $\mathrm{~kW}$ \\
\hline $\bar{S}^{\mathrm{ts}}$ & 30 & $\mathrm{~kW}$ \\
\hline $\begin{array}{c}\underline{l}_{1}^{\mathrm{el}}, \underline{l}_{6}^{\mathrm{el}} \\
(12: 00-15: 59)\end{array}$ & 0.4 & $\mathrm{kWh}$ \\
\hline $\begin{array}{c}\underline{l}_{1}^{\mathrm{el}}, \underline{l}_{6}^{\mathrm{el}} \\
(16: 00-11: 59)\end{array}$ & 0.1 & $\mathrm{kWh}$ \\
\hline $\bar{l}_{1}^{\mathrm{el}}, \bar{l}_{6}^{\mathrm{el}}$ & 0.6 & $\mathrm{kWh}$ \\
\hline$L_{1}^{e l}, L_{6}^{e l}$ & 7.5 & $\mathrm{~kW}$ \\
\hline$\underline{l}_{2}^{\mathrm{el}}, \underline{l}_{7}^{\mathrm{el}}$ & 0.15 & $\mathrm{kWh}$ \\
\hline $\bar{l}_{2}^{\mathrm{el}}, \bar{l}_{3}^{\mathrm{el}}, \bar{l}_{4}^{\mathrm{el}}$, \\
$\bar{l}_{7}^{\mathrm{el}}, \bar{l}_{8}^{\mathrm{el}}, \bar{l}_{9}^{\mathrm{el}}$ & 0.5 & $\mathrm{kWh}$ \\
\hline$L_{2}^{\mathrm{el}}, L_{7}^{\mathrm{el}}$ & 2.5 & $\mathrm{~kW}$ \\
\hline $\begin{array}{c}\underline{l}_{3}^{\mathrm{el}}, \underline{l}_{8}^{\mathrm{el}} \\
(12: 00-15: 59)\end{array}$ & 0.2 & $\mathrm{kWh}$ \\
\hline $\begin{array}{c}\underline{l}_{3}^{\mathrm{el}}, \underline{l}_{8}^{\mathrm{el}} \\
(1600-11: 59)\end{array}$ & 0.1 & $\mathrm{kWh}$ \\
\hline$L_{3}^{\mathrm{el}}, L_{8}^{\mathrm{el}}$ & 6 & $\mathrm{~kW}$ \\
\hline$\underline{l}_{4}^{\mathrm{el}}, \underline{l}_{9}^{\mathrm{el}}$ & 0 & $\mathrm{kWh}$ \\
\hline$L_{4}^{\mathrm{el}}, L_{9}^{\mathrm{el}}$ & 4 & $\mathrm{~kW}$ \\
\hline$\underline{l}_{5}^{\mathrm{el}}, \underline{e}_{10}^{\mathrm{el}}$ & 0.2 & $\mathrm{kWh}$ \\
\hline $\bar{l}_{5}^{\mathrm{el}}, \bar{l}_{10}^{\mathrm{el}}$ & 0.7 & $\mathrm{kWh}$ \\
\hline$L_{5}^{\mathrm{el}}, L_{10}^{\mathrm{el}}$ & 5 & $\mathrm{~kW}$ \\
\hline
\end{tabular}

TABLE II

VALUES OF PERFORMANCE INDICES FOR DIFFERENT ROBUSTNESS FACTORS.

\begin{tabular}{|c|c|c|c|}
\hline Index & Case 1 & Case 2 & Case 3 \\
\hline EC [€] & 5582 & 6409 & 6168 \\
\hline SS & 0.88 & 1 & 0.95 \\
\hline FESR & 0.93 & 0.82 & 0.88 \\
\hline EI & 0.42 & 0.29 & 0.32 \\
\hline PoR [\%] & 0 & 14.9 & 8.9 \\
\hline CVR [\%] & 35.7 & 0 & 9.9 \\
\hline Runtime [s] & 102.8 & 122.1 & 113.3 \\
\hline
\end{tabular}

and $\hat{\nu}(k))$ is determined as $15 \%$ of the nominal values $\left(\kappa_{+}(k)\right.$, $\kappa_{-}(k)$, and $\left.\nu(k)\right)$ for each time slot.

The proposed robust control scheme is implemented in MATLAB R2020a on a desktop PC (i7-7500U core $2.70 \mathrm{GHz}$ processor and 16 GB RAM memory) equipped with the Gurobi optimizer [46]. In the considered setting the MILP problem (83)-(98) is characterized by 961 real and 120 binary decision variables, 1922 bounding constraints, 442 equality constraints, 720 inequality constraints, and 288 integrality constraints.

\section{B. Results Analysis and Discussion}

As a first outcome, the proposed control algorithm is tested on three scenarios that allow to assess the performance of the whole microgrid, and the impact of the budgets of uncertainty on the conservativeness and optimality of the achieved results. The scenarios are defined as follows:

- Case 1 - the deterministic MPC, corresponding to zerovalued budgets of uncertainty (i.e., $\gamma_{q}=\gamma_{d}=\gamma_{\pi}=0$ ). The nominal values are assigned to the uncertain parameters; hence, the protection functions for the objective function and constraints are removed (i.e., $\beta\left(\boldsymbol{\xi}(k), \gamma_{\pi}\right)=$ 0 and $\left.\boldsymbol{\psi}\left(\gamma_{q}\right)=\boldsymbol{\varphi}\left(\gamma_{q}\right)=\mathbf{0}_{H, 1}\right)$.
- Case 2 - the most conservative case, corresponding to the maximum budgets of uncertainty (i.e., $\gamma_{q}=\gamma_{d}=H$ and $\left.\gamma_{\pi}=4 H\right)$. The worst-case realization of uncertain parameters is considered; hence, functions $\beta\left(\boldsymbol{\xi}(k), \gamma_{\pi}\right)$, $\boldsymbol{\psi}\left(\gamma_{q}\right)$, and $\boldsymbol{\varphi}\left(\gamma_{q}\right)$ provide the full protection against data uncertainty.

- Case 3 - the RMPC based on the cardinality uncertainty set, corresponding to a potential choice for the budgets of uncertainty $\gamma_{q}=\gamma_{q}^{*}, \gamma_{d}=\gamma_{d}^{*}$, and $\gamma_{\pi}=\gamma_{\pi}^{*}$ with $\gamma_{q}^{*}, \gamma_{d}^{*} \in$ $(0, H)$ and $\gamma_{\pi}^{*} \in(0,4 H)$, which make the robustness of the solution not significantly changing for $\gamma_{q} \geq \gamma_{q}^{*}$, $\gamma_{d} \geq \gamma_{d}^{*}$, and $\gamma_{\pi} \geq \gamma_{\pi}^{*}$. These values are determined through a sensitivity analyses over different robustness factors (we set $\gamma_{q}^{*}=13, \gamma_{d}^{*}=13$, and $\gamma_{\pi}^{*}=20$ ).

The values of the microgrid performance indices obtained over the whole 2018 under the above defined three scenarios are reported in Table II. As expected, the solution of Case 1 leads to the minimum EC and the best performance for SS, FESR, and EI, even though this corresponds to the most optimistic situation where no effect of uncertainty is addressed. As a consequence, in real conditions, any forecast variation in the nominal profiles of the thermal energy demand, electrical demand, RES generation, and electricity and gas pricing coefficients may produce a significant increase in the achieved value of the objective function (i.e., the EC). Morever, the electricity and gas network constraints are not always satisfied - as shown by the CVR equal to $35.7 \%$ - due to the absence of mitigation of the data uncertainty.

Conversely, the solution of Case 2 ensures full protection against uncertainty. As expected, on the one hand, this implies that constraints are never violated $(\mathrm{CVR}=0)$; on the other hand, this leads to the highest PoR (equal to 14.9\%) since the corresponding highest level of conservativeness is achieved at the expense of maximum distancing from optimality.

Finally, in Case 3 a tradeoff solution is obtained. From the optimality perspective, a slight decrease in the EC as well as in SS, FESR, and EI is apparent; not surprisingly, the PoR (equal to $8.9 \%$ ) is quite lower than the Case 2. At the same time, the solution of Case 3 is quite protected against uncertainty, implying a CVR equal to $9.9 \%$. This confirms the effectiveness of the RMPC algorithm to determine a practical compromise between the microgrid perfomance specified by the optimization objective (e.g., the EC) and the protection against the constraint violation.

Furthermore, the last row of Table II reports the average runtime in the three cases: the computational time over all the simulations is around two minutes.

As a second outcome, we present a sensitivity analysis of the 10000 runs $\mathrm{MC}$ simulation results with respect to different budgets of uncertainty $\gamma_{q}=\gamma_{d} \in[0, H]$ and $\gamma_{\pi} \in[0,4 H]$ in terms of average PoR and CVR, all reported in Fig. 2. As can be observed from the results, both the PoR and CVR present a non-linear trend. On the one hand, for any fixed value of $\gamma_{\pi}$, as the value of $\gamma_{q}=\gamma_{d}$ increases, the PoR monotonically gets worse, whilst the CVR monotonically gets better. On the other hand, for a fixed high value of $\gamma_{q}=\gamma_{d}$, both the PoR and CVR are quite constant with respect to changes in $\gamma_{\pi}$; conversely, for a fixed low value of $\gamma_{q}=\gamma_{d}$, the variations of the PoR 
(a)

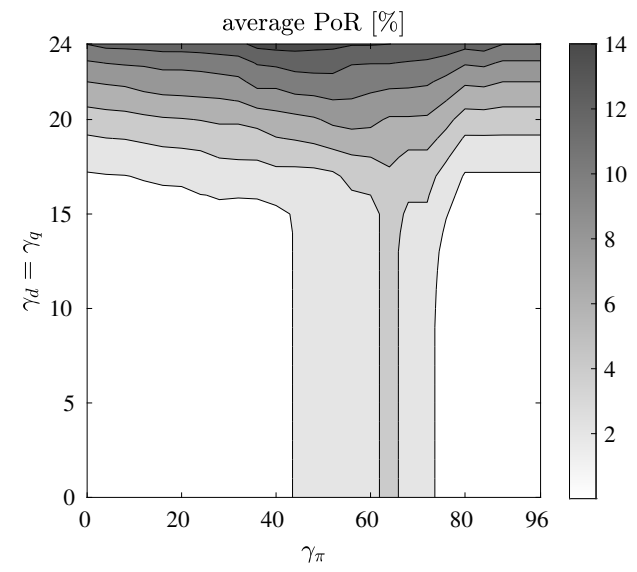

(b)

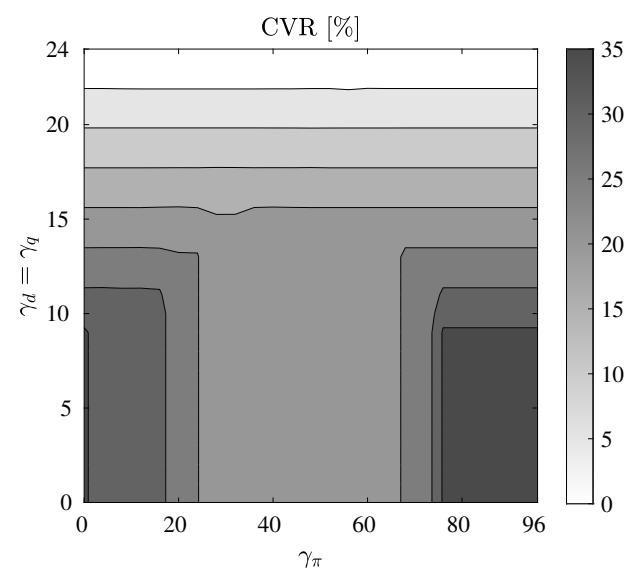

Fig. 2. Sensitivity analysis of the average PoR (a) and CVR (b) with respect to different budgets of uncertainty.

and CVR have a convex and concave profile presenting a local maximum and minimum, respectively. In addition, the PoR and the CVR present a mutually dual behavior, confirming that they are two competing indices: the PoR is lower where the CVR is higher, and viceversa. This result confirms the effectiveness of our approach, enabling the chance of a good trade-off between the total energy payment and the level of conservativeness by changing the value of the budget of uncertainty.

\section{Comparison with a Baseline Robust Method}

With the aim of evaluating and better highlighting the advantages of our approach with respect to the related literature, we provide a comparison between the results obtained by the proposed technique and those achieved by a baseline method, namely the robust approach based on the box-uncertainty-set. Such a method relies on the assumption that uncertain parameters take values from their own different ranges independently, as indicated in [36], [47].

Specifically, we report the findings of a sensitivity analysis based on the MC simulation, where the microgrid performance indices and level of conservativeness of the energy scheduling strategies is determined for both the proposed (i.e., RMPC based on cardinality-uncertainty-set) and the (a)

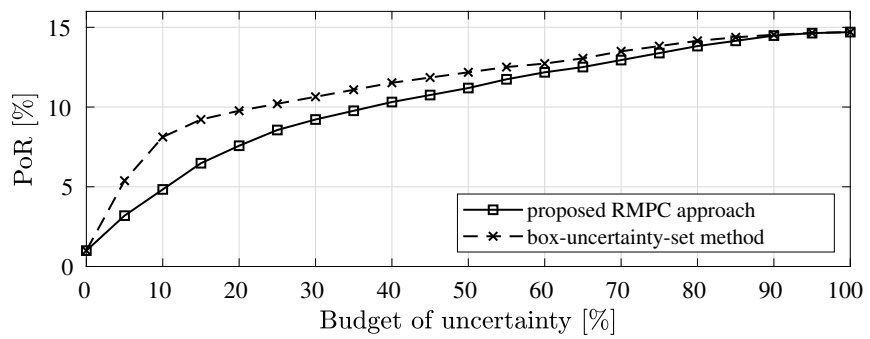

(b)

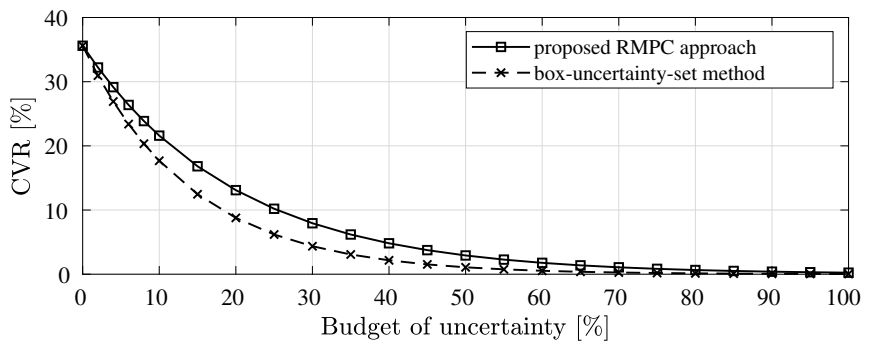

Fig. 3. PoR (a) and CVR (b) with respect to different budgets of uncertainty for the RMPC based on cardinality- and box-uncertainty-set.

baseline method (i.e., RMPC based on box-uncertainty-set) for different budgets of uncertainty. In Fig. 3 we show the results of the comparison analysis in terms of PoR and CVR. It is apparent that, event though the CVR of the proposed RMPC is higher than the box-uncertainty-set method for small budgets of uncertainty, the proposed RMPC always offers a less conservative solution. In fact, as shown in Fig. 3.a, the PoR of the proposed RMPC exhibits lower values than the box-uncertainty-set method. In addition, from medium to large budgets of uncertainty, the CVR of the proposed RMPC is comparable to the box-uncertainty-set method (Fig. 3.b).

These results corroborate the effectiveness of the RMPC based on cardinality-uncertainty-set: a good compromise between the total energy cost and the level of conservativeness can be effectively achieved by the microgrid manager by suitably tuning the value of the budget of uncertainty.

\section{CONCLUSiOnS AND Future WORK}

This research work proposes a novel robust model predictive control algorithm for microgrids that comprise noncontrollable and controllable thermal and electrical loads, i.e., multi-carrier microgrids. The aim of this work is to take advantage of the robust MPC approach in the context of multicarrier microgrids, where contributions are still particularly limited. The objective of the proposed control strategy is to minimize the overall economical cost and the energy flow from the main grid, while ensuring thermal comfort. Moreover, thanks to the robustness of the method, uncertainty in the system model can be addressed, such as disturbances on loads, on renewable energy production, and on electrical and thermal need. The effectiveness of the proposed method is proved by simulations results obtained for a Dutch residential building and based on real data. The tests show a substantial decrease in the electric and thermal balance breach when compared to the outcomes of the deterministic MPC. 
Finally, several remarks are in order. From the control perspective, the proposed approach relies on a centralized control architecture, that generally could suffer from scalability issues. Hence, future development will focus on extending the proposed algorithm to a decentralized and distributed setting and investigating the corresponding performance in large scale scenarios. As for the system modeling, one may observe that results and implications are derived relying on some assumptions such as the price inelasticity. Actually, this limitation is only apparent, since the proposed model can be easily generalized to more complex cases by incorporating different types of objective functions and constraints. An interesting development of our research will be devoted to integrating nonlinear stepwise function to better model the price of both electricity and gas. Moreover, we will consider expanding our current model with start-up and shut-down cost and timing constraints related to thermal and electrical generation. Future researchs will also consider further types of uncertainty that could impact decision parameters and regard the outflow of the thermal energy from the microgrid to the district in exchange for economical compensation. Finally, from the methodological point of view, since the proposed approach concerns an online multistage decision problem, it would be interesting to implement a multistage robust optimization method and comparing the resulting performance both in terms of optimality and of computational tractability.

\section{REFERENCES}

[1] X. Fang, S. Misra, G. Xue, and D. Yang. Smart grid - the new and improved power grid: A survey. IEEE Commun. Surveys Tuts, 14(4):944-980, 2012.

[2] A. Hirsch, Y. Parag, and J. Guerrero. Microgrids: A review of technologies, key drivers, and outstanding issues. Renew. Sust. Energ. Rev., 90:402-411, 2018.

[3] B. Yan, P. B. Luh, G. Warner, and P. Zhang. Operation and design optimization of microgrids with renewables. IEEE Trans. Autom. Sci. Eng., 14(2):573-585, 2017.

[4] Y. Lan, X. Guan, and J. Wu. Online decentralized and cooperative dispatch for multi-microgrids. IEEE Trans. Autom. Sci. Eng., 17(1):450 462, 2019.

[5] R. Minciardi and M. Robba. A bilevel approach for the stochastic optimal operation of interconnected microgrids. IEEE Trans. Autom. Sci. Eng., 14(2):482-493, 2016.

[6] Z. Yang, L. Xia, and X. Guan. Fluctuation reduction of wind power and sizing of battery energy storage systems in microgrids. IEEE Trans. Autom. Sci. Eng., 17(3):1195-1207, 2020.

[7] R. Carli, M. Dotoli, J. Jantzen, M. Kristensen, and S. Ben Othman. Energy scheduling of a smart microgrid with shared photovoltaic panels and storage: The case of the Ballen marina in Samsø. Energy, 198:117188, 2020.

[8] T. Pippia, J. Lago, R. De Coninck, and B. De Schutter. Scenario-based nonlinear model predictive control for building heating systems. arXiv eprint 2012.02011, 2020.

[9] E. F. Camacho and C. B. Alba. Model Predictive Control. Advanced Textbooks in Control and Signal Processing. Springer London, 2013.

[10] D. Q. Mayne. Model predictive control: Recent developments and future promise. Automatica, 50(12):2967-2986, 2014.

[11] A. Parisio, M. Molinari, D. Varagnolo, and K. H. Johansson. A scenariobased predictive control approach to building HVAC management systems. In 2013 IEEE International Conference on Automation Science and Engineering (CASE), pages 428-435, 2013.

[12] H. Bittel, C. N. Jones, and A. Parisio. Use of Model Predictive Control for Short-Term Operating Reserve Using Commercial Buildings in the United Kingdom Context. In 2018 IEEE Decis. Contr. P., pages 73087313, 2018.
[13] Z. Chen, L. Wu, and Y. Fu. Real-time price-based demand response management for residential appliances via stochastic optimization and robust optimization. IEEE Transactions on Smart Grid, 3(4):1822-1831, 2012.

[14] A. Parisio, E. Rikos, and L. Glielmo. A model predictive control approach to microgrid operation optimization. IEEE Trans. Control Syst. Technol., 22(5):1813-1827, 2014.

[15] F. Verrilli, S. Srinivasan, G. Gambino, M. Canelli, M. Himanka, C. Del Vecchio, M. Sasso, and L. Glielmo. Model predictive control-based optimal operations of district heating system with thermal energy storage and flexible loads. IEEE Trans. Autom. Sci. Eng., 14(2):547-557, 2017.

[16] A. Parisio, E. Rikos, and L. Glielmo. Stochastic model predictive control for economic/environmental operation management of microgrids: An experimental case study. J. Process Contr., 43:24-37, 2016.

[17] F. Liberati, A. Di Giorgio, A. Giuseppi, A. Pietrabissa, E. Habib, and L. Martirano. Joint model predictive control of electric and heating resources in a smart building. IEEE Trans. Ind Appl., 55(6):7015-7027, 2019.

[18] Y. Zhang, R. Wang, T. Zhang, Y. Liu, and B. Guo. Model predictive control-based operation management for a residential microgrid with considering forecast uncertainties and demand response strategies. IET Generation, Transmission Distribution, 10(10):2367-2378, 2016.

[19] T. Wakui, K. Sawada, R. Yokoyama, and H. Aki. Predictive management for energy supply networks using photovoltaics, heat pumps, and battery by two-stage stochastic programming and rule-based control. Energy, 179:1302-1319, 2019.

[20] R. Carli, G. Cavone, T. Pippia, B. De Schutter, and M. Dotoli. A robust mpc energy scheduling strategy for multi-carrier microgrids. In 2020 IEEE 16th International Conference on Automation Science and Engineering (CASE), pages 152-158, 2020.

[21] N. Holjevac, T. Capuder, N. Zhang, I. Kuzle, and C. Kang. Corrective receding horizon scheduling of flexible distributed multi-energy microgrids. Applied Energy, 207:176-194, 2017.

[22] J. Vasilj, D. Jakus, and P. Sarajcev. Robust nonlinear economic mpc based management of a multi energy microgrid. IEEE Transactions on Energy Conversion, 2020.

[23] F. Alavi, E. Park Lee, N. van de Wouw, B. De Schutter, and Z. Lukszo. Fuel cell cars in a microgrid for synergies between hydrogen and electricity networks. Appl. Energ., 192:296-304, 2017.

[24] M. Pereira, D. Muñoz de la Peña, and D. Limon. Robust economic model predictive control of a community micro-grid. Renew. Energ., 100:3-17, 2017. Special Issue: Control and Optimization of Renewable Energy Systems.

[25] Y. Zhang, L. Fu, W. Zhu, X. Bao, and C. Liu. Robust model predictive control for optimal energy management of island microgrids with uncertainties. Energy, 164:1229-1241, 2018.

[26] M. Zhai, Y. Liu, T. Zhang, and Y. Zhang. Robust model predictive control for energy management of isolated microgrids. In 2017 IEEE In. C. Ind. Eng. Eng. Man., pages 2049-2053, 2017.

[27] L. G. Marín, M. Sumner, D. Muñoz-Carpintero, D. Köbrich, S. Pholboon, D. Sáez, and A. Núñez. Hierarchical energy management system for microgrid operation based on robust model predictive control. Energies, 12(23), 2019.

[28] D. Bertsimas and M. Sim. The price of robustness. Operations research, 52(1):35-53, 2004.

[29] I. Staffell, D. Brett, N. Brandon, and A. Hawkes. A review of domestic heat pumps. Energy \& Environmental Science, 5(11):9291-9306, 2012.

[30] R. Hermansen, K. Smith, J. E. Thorsen, J. Wang, and Y. Zong. Model predictive control for a heat booster substation in ultra low temperature district heating systems. Energy, 238:121631, 2022.

[31] B. Mayer, M. Killian, and M. Kozek. A branch and bound approach for building cooling supply control with hybrid model predictive control. Energy and Buildings, 128:553-566, 2016.

[32] N. M. Isa, C. W. Tan, and A. H. M. Yatim. A comprehensive review of cogeneration system in a microgrid: A perspective from architecture and operating system. Renewable and Sustainable Energy Reviews, 81:2236$2263,2018$.

[33] G. K. H. Larsen, N. D. van Foreest, and J. M. A. Scherpen. Distributed mpc applied to a network of households with micro-chp and heat storage. IEEE Transactions on Smart Grid, 5(4):2106-2114, 2014.

[34] Y. Zhang, F. Meng, R. Wang, B. Kazemtabrizi, and J. Shi. Uncertaintyresistant stochastic MPC approach for optimal operation of CHP microgrid. Energy, 179:1265-1278, 2019.

[35] A. Bemporad and M. Morari. Control of systems integrating logic, dynamics, and constraints. Automatica, 35(3):407-427, 1999. 
[36] A. L. Soyster. Convex programming with set-inclusive constraints and applications to inexact linear programming. Operations research, 21(5):1154-1157, 1973.

[37] B. L. Gorissen, I. Yanıkoğlu, and D. den Hertog. A practical guide to robust optimization. Omega, 53:124-137, 2015.

[38] D. Bertsimas, D. B. Brown, and C. Caramanis. Theory and applications of robust optimization. SIAM review, 53(3):464-501, 2011.

[39] E. Delage and D. A. Iancu. Robust multistage decision making. In The operations research revolution, pages 20-46. INFORMS, 2015.

[40] S. R. Cominesi, M. Farina, L. Giulioni, B. Picasso, and R. Scattolini. A two-layer stochastic model predictive control scheme for microgrids. IEEE Transactions on Control Systems Technology, 26(1):1-13, 2017.

[41] D. E. Olivares, C. A. Cañizares, and M. Kazerani. A centralized energy management system for isolated microgrids. IEEE Transactions on smart grid, 5(4):1864-1875, 2014.

[42] Neon Neue Energieökonomik, Technical University of Berlin, ETH Zürich, and DIW Berlin. Open-power-system-data. https://data. open-power-system-data.org/.

[43] European Commission SETIS. Emhires dataset solarpower. https://setis.ec.europa.eu/publications/relevant-reports/ emhires-dataset-part-ii-solarpower-generation.

[44] Nord Pool. Day-ahead prices. https://www.nordpoolgroup.com/ Market-data1/Dayahead/Area-Prices/ALL1/Hourly/?view=table.

[45] MEKH \& VaasaETT Energie-Control. Energy price index. https://www. energypriceindex.com/latest-update/.

[46] Gurobi Optimization, Inc. Gurobi optimizer reference manual. https: //www.gurobi.com, 2016.

[47] T. Hubert and S. Grijalva. Modeling for residential electricity optimization in dynamic pricing environments. IEEE Transactions on Smart Grid, 3(4):2224-2231, 2012.

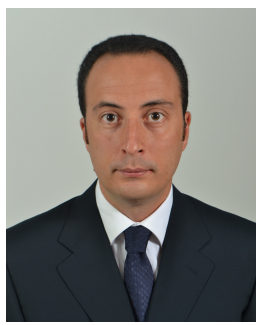

Raffaele Carli (M'17) received the Laurea degree in Electronic Engineering with honours in 2002 and the Ph.D. in Electrical and Information Engineering in 2016, both from Politecnico di Bari, Italy. From 2003 to 2004, he was a Reserve Officer with Italian Navy. From 2004 to 2012, he worked as System and Control Engineer and Technical Manager for a space and defense multinational company.

Dr. Carli is currently an Assistant Professor in Automatic Control at Politecnico di Bari, and his research interests include the formalization, simulation, and implementation of decision and control systems, as well as modeling and optimization of complex systems. He was member of the International Program Committee of 20+ international conferences and guest editor for special issues on international journals. He is author of $60+$ printed international publications.

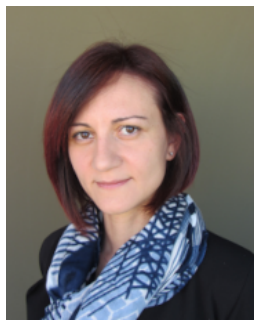

Graziana Cavone (S'16, M'18) received the Laurea degree summa cum laude in Control Engineering in 2013 from the Polytechnic of Bari, Italy and the $\mathrm{Ph} . \mathrm{D}$. degree (excellent with honors) in Electronic and Computer Engineering in 2018 from the University of Cagliari, Italy. She currently is a postdoc Research Fellow at Polytechnic of Bari. She has been Research Fellow in 2014 at Polytechnic of Bari, Italy, and Visiting Ph.D. Student in 2016-2017 at Delft University of Technology, the Netherlands. Her research interests include modelling, simulation, optimization, and control of discrete-event and hybrid systems, distributed control, automated manufacturing systems, intelligent transportation, smart cities. She is the Local Arrangements chair of the 2021 Mediterranean Conference on Control and Automation. She is Associate Editor for the international Journal Results in Control and Optimization (RICO). She was member of the International Program Committee of 20+ international conferences and Guest Editor for special issues on international journals. She was awarded a research grant by the National Science Foundation of China for year 2020.

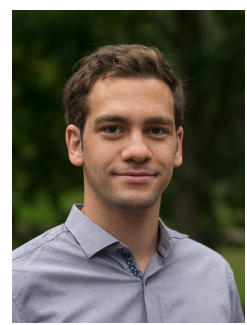

Tomás Pippia received the B.Sc. degree in Electronic Engineering and the M.Sc. degree in Systems and Control Engineering from the University of Pavia, Italy, in 2013 and 2015, respectively. He received the Ph.D. degree in Systems and Control from the Delft University of Technology, The Netherlands. He is currently a Postdoctoral Researcher at the Delft University of Technology working on distributed control for constellation of satellites in partnership with the European Space Agency.

His current research interests include smart grids, model-based control, and distributed satellite and spacecraft control.

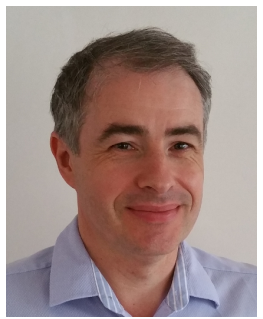

Bart De Schutter (M'08-SM'10-F'19) received the PhD degree in Applied Sciences (summa cum laude with congratulations of the examination jury) in 1996, at K.U. Leuven, Belgium. Currently, he is a Full Professor and Head of Department at the Delft Center for Systems and Control of Delft University of Technology in Delft, The Netherlands.

Prof. De Schutter is a Senior Editor of the IEEE Transactions on Intelligent Transportation Systems and an Associate Editor of IEEE Transactions on Automatic Control. His current research interests include multi-level and multi-agent control, optimization-based control, learning-based control, control of hybrid systems with applications in intelligent transportation systems and smart energy systems.

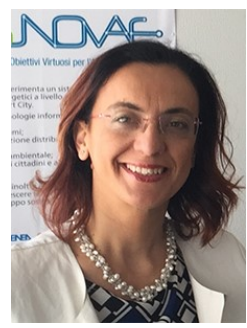

Mariagrazia Dotoli (M'96, SM'12) received the Laurea degree in Electronic Engineering with honours in 1995 and the Ph.D. in electrical engineering in 1999 from Politecnico di Bari (Italy).

She has been a visiting scholar at the Paris 6 University and at the Technical University of Denmark. She is expert evaluator of the European Commission since the 6th Framework Programme. She is a Full Professor in Automatic Control at Politecnico di Bari, which she joined in 1999. She has been the Vice Rector for research of Politecnico di Bari and a member elect of the Academic Senate. Her research interests include modeling, identification, management, control and diagnosis of discrete event systems, manufacturing systems, logistics systems, traffic networks, smart grids and networked systems.

Prof. Dotoli was co-chairman of the Training and Education Committee of ERUDIT, the European Commission network of excellence for fuzzy logic and uncertainty modeling in information technology, and was key node representative of EUNITE, the EUropean Network of excellence on Intelligent Technologies. She is a Senior Editor of the IEEE TRANS. ON AUTOMATION SCIENCE AND ENGINEERING and an Associate Editor of the IEEE TRANS. ON SYSTEMS, MAN, AND CYBERNETICS and of the IEEE TRANS. ON CONTROL SYSTEMS TECHNOLOGY. She is the General chair of the 2021 Mediterranean Conference on Control and Automation. She was the Program chair of the 2020 IEEE Conference on Automation Science and Engineering, the Program co-chair of the 2017 IEEE Conference on Automation Science and Engineering, the Workshop and Tutorial chair of the 2015 IEEE Conference on Automation Science and Engineering, the Special Session co-chair of the 2013 IEEE Conference on Emerging Technology and Factory Automation and chair of the National Committee of the 2009 IFAC Workshop on Dependable Control of Discrete Systems. She was member of the International Program Committee of 80+ international conferences. She is author of $200+$ publications, including 1 textbook (in Italian) and 70+ international journal papers. 